\title{
Convex Sensing-Reporting Optimization for Cooperative Spectrum Sensing
}

\author{
by \\ Adam Noel \\ B.Eng, Memorial University of Newfoundland, 2009

\begin{abstract}
A THESIS SUBMITTED IN PARTIAL FULFILLMENT
OF THE REQUIREMENTS FOR THE DEGREE OF

Master of Applied Science
\end{abstract} \\ in \\ THE FACULTY OF GRADUATE STUDIES \\ (Electrical and Computer Engineering) \\ The University Of British Columbia \\ (Vancouver) \\ August 2011 \\ (c) Adam Noel, 2011
}




\section{Abstract}

In this thesis, we consider the cooperative spectrum sensing problem in cognitive radio with energy detection. Secondary users with non-identical, independent sensing channels make 1-bit sensing decisions and report their decisions to the secondary base station over orthogonal noisy fading channels. The base station has knowledge of the reporting channel coefficients and acts as a fusion center by combining the decisions with an $M$-out-of- $K$ rule. We allow the secondary users to trade sensing time slots for additional reporting time slots to increase the signalto-noise ratios of the reporting channels. We derive the corresponding false alarm and missed detection probabilities, which are functions of the secondary sensor decision thresholds and the durations for sensing and reporting. Furthermore, we bound these probabilities and impose a practical convex region to enable the application of convex optimization for minimization of the false alarm probability for a target missed detection probability. We consider the two cases where the instantaneous and the average reporting channels are known for optimization. Allowing secondary users to trade sensing time slots for additional reporting time slots is shown to significantly improve sensing performance in both cases, even with poor sensing channels and a small number of secondary users. 


\section{Preface}

Some of the content in this thesis has previously been accepted for publication. Specifically,

- A. Noel and R. Schober, "Convex sensing-reporting optimization for cooperative spectrum sensing," Proceedings of IEEE PIMRC, September 2011. Content contained in this publication includes portions of all Chapters, except for most of Section 1.1, Section 3.3, most of Section 5.1, and the portions of Chapters 4 and 5 that discuss fusion rules other than OR-rule.

I am the primary author for the publication above and have performed the majority of the work. My tasks included but were not limited to literature review, analytical derivations, simulation coding and execution, and writing of the manuscripts. My supervisor, listed as the second author above, provided invaluable help and guidance in a secondary role.

The IEEE holds the copyright for the publication above. However, I retain the right to extract material verbatim from the work for the publication of my thesis. 


\section{Table of Contents}

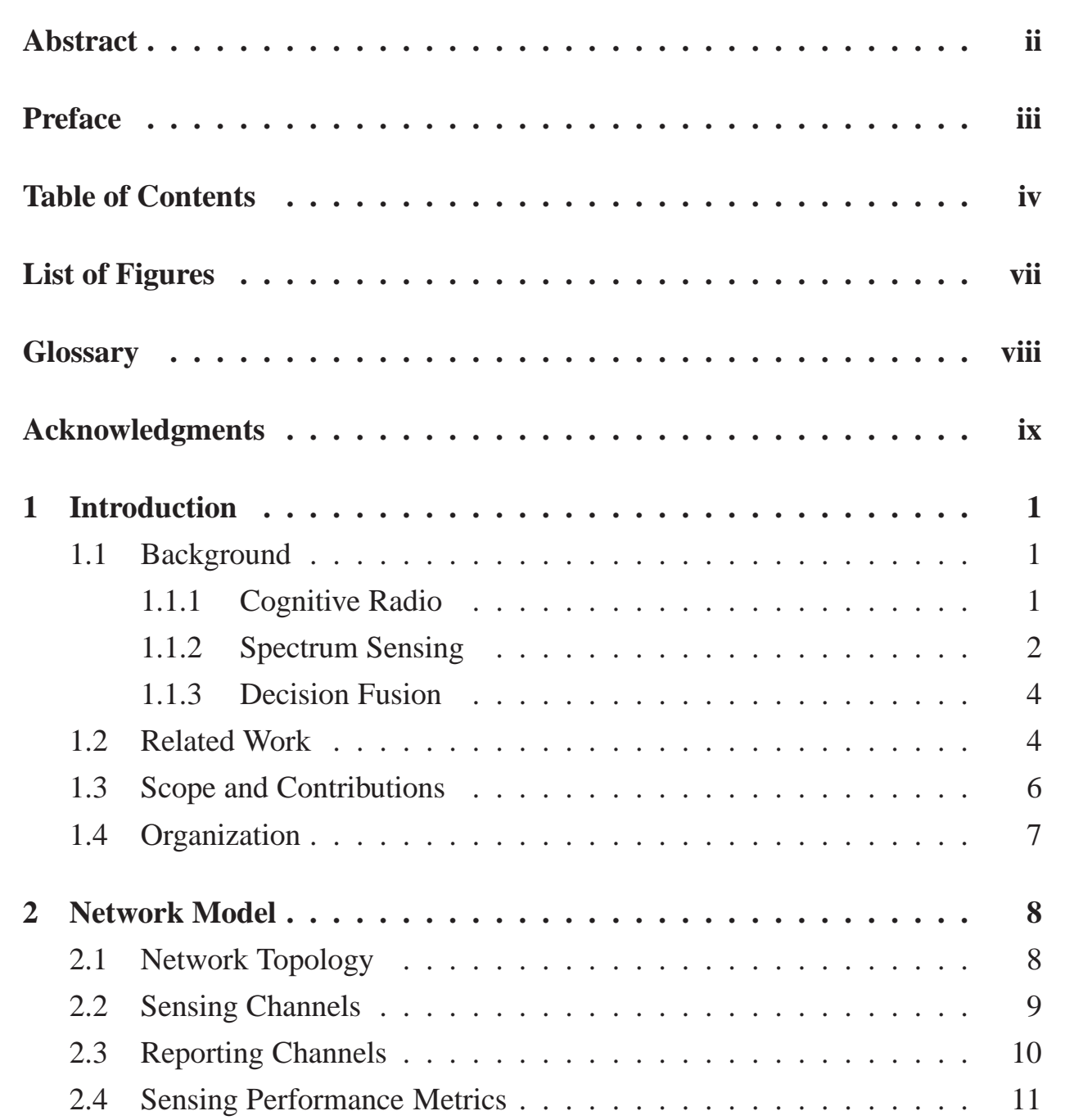


3 Single-Sensor Network $\ldots \ldots \ldots \ldots \ldots$. . . . . . . . . 12

3.1 FC Performance . . . . . . . . . . . . . . . . . . . . . . . . . . . . . . .

$3.1 .1 \quad$ Local Sensing . . . . . . . . . . . . . . . . . . 13

3.1 .2 Sensor Reporting . . . . . . . . . . . . . . . . . 14

3.2 Closed-Form, Convex Bounds on Performance . . . . . . . . 15

3.2.1 Upper Bounds on Performance . . . . . . . . . . . . . 15

3.2.2 Convexity of Upper Bounds . . . . . . . . . . . . 17

3.3 Performance Averaged Over Reporting Channels . . . . . . . . . 18

3.4 Single-Sensor Optimization Problem . . . . . . . . . . . . . . 19

4 Multi-Sensor Network ..................... 21

4.1 Optimization Problem . . . . . . . . . . . . . 22

4.1.1 Exact Expressions for Decision Fusion . . . . . . . . 22

4.1.2 Exact Expressions and Bounds for Decision Fusion . . . . 23

4.1.3 Bounds Applied to OR-Rule . . . . . . . . . . . . . 24

4.1.4 Generalized Convex Multiplicative Programming . . . . . 24

4.2 Suboptimal Convex Problem for OR-Rule . . . . . . . . . . 25

4.3 Suboptimal Convex Problem for Generalized Counting Rule . . . 27

5 Numerical Results and Discussion $\ldots \ldots \ldots$. . . . . . . . 28

5.1 Single-Sensor Results . . . . . . . . . . . . . . . . . . . . . . 29

5.2 Multi-Sensor Results and Discussion . . . . . . . . . . . . . . . . . 32

5.2.1 Comparison of Fusion Rules . . . . . . . . . . . . . . 32

5.2.2 Sensitivity to Channel Quality with OR-Rule . . . . . . . 34

5.2.3 Sensitivity to Channel Quality with Other Rules . . . . . . 36

6 Conclusions and Future Work .................. 39

6.1 Conclusions . . . . . . . . . . . . . . . . . . . 39

6.2 Future Work . . . . . . . . . . . . . . . . 40

Bibliography ......................... 41

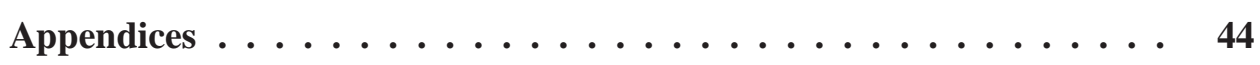

A Proof of Theorem $1 \ldots \ldots \ldots \ldots \ldots$ 
B Proof of Convexity of Bound on $(3.16) \ldots \ldots$

C Proof of Convexity of Bound on $(3.15) \ldots \ldots \ldots$

D Proof of Theorem $3 \ldots \ldots \ldots \ldots$

E Proof of Theorem $4 \ldots \ldots \ldots \ldots \ldots$ 


\section{List of Figures}

Figure 2.1 Secondary network near a primary transmitter. . . . . . . . 9

Figure 2.2 Division of sensing and reporting time slots for the $k$ th sensor. $\quad 10$

Figure 5.1 ROC for $\gamma_{R, k}=-6 \mathrm{~dB}$ with 1 sensor and three different values of $N \ldots \ldots \ldots \ldots \ldots \ldots \ldots \ldots$

Figure 5.2 ROC for $\bar{\gamma}_{R, k}=-6 \mathrm{~dB}$ with 1 sensor and $N=5000 \ldots \ldots . .31$

Figure 5.3 Secondary network ROC with 4 sensors and $N=5000 \ldots 33$

Figure 5.4 Effect of sensing channels on $P_{f F C}$ with 4 sensors using ORrule and $P_{m F C_{T A R}}=0.005 . \ldots \ldots \ldots \ldots$

Figure 5.5 Effect of reporting channels on $P_{f F C}$ with 4 sensors and $N=$ 5000, using OR-rule, and $P_{m F C_{T A R}}=0.005 . \ldots \ldots \ldots$

Figure 5.6 Effect of sensing channels on $P_{f F C}$ with 4 sensors using $M=2$ and $P_{m F C_{T A R}}=0.005 \ldots \ldots \ldots \ldots \ldots \ldots$

Figure 5.7 Effect of reporting channels on $P_{f F C}$ with 4 sensors and $N=$ 5000, using $M=2$, and $P_{m F C_{T A R}}=0.005 \ldots \ldots \ldots$ 


\section{Glossary}

The following are abbreviations and acronyms used in this thesis, listed in alphabetical order.

AWGN Additive White Gaussian Noise

BPSK Binary Phase-Shift Keying

FC Fusion Center

IEEE The Institute of Electrical and Electronics Engineers

PU Primary User

ROC Receiver Operating Characteristic

SNR Signal-to-Noise Ratio

SU Secondary User 


\section{Acknowledgments}

My sincerest thanks go to my supervisor, Prof. Robert Schober, whose continued patience and advice made this thesis possible.

Funding for this thesis was provided by the Natural Sciences and Engineering Research Council of Canada, as well as the University of British Columbia.

I owe many thanks for the support and encouragement of my family. My parents Josiah and Pauline have been the pillars of my life, even as I have pursued my education "on the Mainland." My wife Joanne has been particularly supportive, even though she will claim (and I will agree) that our wedding was the most important accomplishment this year. 


\section{Chapter 1}

\section{Introduction}

\subsection{Background}

\subsubsection{Cognitive Radio}

The introduction of cognitive radio in [1] set the foundation for what has become a very active research area for wireless communications in the last decade. Cognitive radio envisions the deployment of wireless devices that are sufficiently intelligent to operate autonomously and adaptively.

In [2], two primary objectives for cognitive radio were identified: reliable communication and efficient use of wireless spectrum. The latter objective is often regarded as requiring modifications to the traditional wireless spectrum licensing model to enable implementation. The traditional licensing model is an approach where specific companies or applications are given exclusive rights to a particular frequency band. In practice, many licensed bands are actually unoccupied in either time or space or both. However, it is unreasonable to expect license holders to sacrifice their exclusivity to a band to permit gains in wireless efficiency. If unlicensed wireless users could access spectrum when it is unoccupied by licensed users, i.e., by taking advantage of spectral holes, then a significant increase in wireless spectral efficiency could result while protecting the interests of those who hold licenses. Unlicensed access would require not only major technological advances, but also regulatory approval to condone its use. Regulatory bodies are interested in 
modifying the traditional spectrum access model [3], thereby motivating research to guide these changes.

One standard has already been written to implement aspects of cognitive radio technology in real environments. The Institute of Electrical and Electronics Engineers (IEEE) Standard 802.22 was recently published with the aim of enabling wireless internet access for sparsely populated rural areas using frequency bands licensed to television broadcasters [4]. Publication of the standard means that companies can now build and sell devices that meet the specifications of the standard.

In a cognitive radio context, licensed users (such as television transmitters or cellphones) are called Primary Users (PUs) while unlicensed users are called Secondary Users (SUs) [5]. This naming convention is more general because some applications with rights to a particular frequency band may not hold an actual license, e.g., wireless microphones are also considered incumbents for IEEE Standard 802.22 [4]. Referring to unlicensed users as secondary emphasizes that their access to spectrum is contingent on the existent of a spectral hole.

\subsubsection{Spectrum Sensing}

The monitoring of frequency bands in order to identify the presence of an active transmitter is known as spectrum sensing, and is one of the fundamental problems in cognitive radio [5]. It is particularly critical for scenarios where primary and secondary networks do not communicate directly, and is listed as one of the cognitive radio capabilities required for IEEE Standard 802.22 devices [4]. The correct identification of spectral holes is then dependent on the reliability of the secondary network's spectrum sensing.

There are three main categories of spectrum sensing [6]:

1. Coherent detection with a matched filter can be deployed when the channel realization between the PU and the SU is known. This is not necessarily a realistic assumption because direct communication is normally required in order to learn the instantaneous channel.

2. Cyclostationary feature detectors can identify PUs by analyzing the spectral correlation of the frequency band. Modulated signal energy can be differentiated from uncorrelated noise because of the cyclostationarity embedded in 
the transmitted signal. While accurate, these detectors are computationally complex.

3. Energy detection measures the energy of the received signal at the sensor and makes a decision based on a sensing threshold. This method is preferred when the information known about the PU is limited; the power of the PU signal is sufficient, though this information must be accurate in order for energy detection to be reliable. Nevertheless, energy detection is the most popular method for spectrum sensing due to its low complexity [6-10], and we adopt it for the analysis in this thesis.

Cooperative spectrum sensing, where multiple SUs share sensing data to improve performance in detecting the status of a PU, was first applied to cognitive radio in [5] and has seen significant research interest in the past few years. A recent survey that summarizes the many challenges in applying cooperative spectrum sensing to cognitive radio is found in [6]. SUs act as local sensors that independently assess whether the PU is active or idle. Most literature in this area considers the presence of a secondary base station that receives sensing results reported from the SUs, cf. e.g. [7-16]. The base station then acts as a Fusion Center (FC) by fusing the individual reporting results to obtain a global decision. Detecting the PU as idle indicates the ability to use the PU's frequency band for secondary data transmission, thereby increasing wireless efficiency.

The feasibility of SU operation depends on the false alarm and missed detection probabilities of cooperative spectrum sensing. False alarm occurs when the secondary network declares that the PU is active and it is actually idle. When there is a false alarm, the secondary network is not taking advantage of a legitimate opportunity to transmit. Missed detection occurs when the secondary network declares that the PU is idle and it is actually active. When there is missed detection, the secondary network may attempt transmission and thereby generate undesired interference to the PU. Furthermore, its own transmission may be unsuccessful due to the PU's activity. There is typically a tradeoff between these two probabilities, and while the PU would prefer a low missed detection probability, the secondary network would prefer a low false alarm probability. 


\subsubsection{Decision Fusion}

As noted, the FC is used to combine information received by the sensors to obtain

a global decision. There are two main categories of fusion, depending on what is transmitted by the sensors. If the sensors transmit their raw sensing data or statistics, i.e., the power of the received signal, then the FC can deploy soft combining. If the sensors make a local decision based on their sensing thresholds and transmit their decisions to the FC, then the FC deploys hard combining or decision fusion [6].

Decision fusion is advantageous in a cognitive radio context where we cannot assume that sensors have large reporting bandwidth available; every sensor must report no more than 1 bit of information, so a narrow dedicated channel is sufficient for the secondary network's reporting requirements. Soft combining, however, requires much more bandwidth to sufficiently quantize the sensing results.

Decision fusion can be optimally implemented by the Chair-Varshney rule, which requires previous knowledge of the false alarm and missed detection probabilities associated with each sensor [17]. Simpler methods include counting rules, such that sensors effectively vote with their sensing decisions and the FC uses a vote threshold to make a global decision. The most common counting rules are the OR-rule, where one sensor detecting the PU as active is sufficient for the FC to declare the same, and the AND-rule, where all sensors must declare that the PU is active [17].

\subsection{Related Work}

The derivation of false alarm and missed detection probabilities where the SUs use an $L_{p}$-norm detector and perfect reporting has been recently performed in [18], for which the energy detector is a special case (i.e., $p=1$ ). The missed detection probability was averaged over the Rayleigh-faded sensing channels, since it was assumed that the instantaneous sensing channels were unknown to the SUs. Furthermore, in [7], these probabilities have been derived using energy detectors where band-limited reporting channels are impaired by noise and fading.

In practice, it is of interest to optimize performance by minimizing the false alarm probability given a target missed detection probability (or vice versa). Op- 
timization has previously been performed for cooperative spectrum sensing with perfect reporting, cf. e.g. [8, 9, 11-13].

In [8], network throughput is maximized by optimizing the division of time slots between sensing the PU and performing secondary data transmission. Both soft and hard combining are considered.

In [9], the number of SUs and the SU sensing thresholds are optimized to minimize the total error probability (false alarm plus missed detection), under the assumption that all SUs have identical sensing channels. The analysis determines the optimal counting rule, the optimal local sensing threshold, and the optimal number of users to select for sensing when the number of sensors becomes large.

In [11], throughput is maximized by optimizing the SU sensing thresholds and decision fusion weights. Convexity of the sensing error probabilities is shown with respect to the SU thresholds but the results are for a sensing channel that is known at the base station.

In both [12] and [13], one error probability is minimized with respect to a target for the other. In [12], the decision threshold is optimized for a model where the FC knows the distances between the PU and the SUs. Small-scale fading is ignored, so the analysis is relatively simplified. The FC applies AND-rule and OR-rule. In [13], the number of local sensing operations is optimized, given that each operation has known false alarm and missed detection probabilities. The FC uses OR-rule, and multiple antennas at the SUs enable simultaneous sensing and reporting.

The optimization of spectrum sensing with imperfect reporting channels has usually been performed with the assumption that the reporting channel has unlimited bandwidth, cf. e.g. [14-16]. Furthermore, optimization with limited reporting bandwidth has focussed primarily on OR-rule and AND-rule (cf. e.g. [8, 12, 13]), whereas optimization generalized to $M$-out-of- $K$ rules has assumed that all sensors have identical sensing performance, as in [9]. A realistic physical environment is inhomogeneous, so we can expect that the strength of the sensing and reporting channels vary among the SUs. For example, the relative location of the SUs to both the PU and base station, and the placement of obstacles, can contribute to channel strength. Thus, it is of interest to optimize scenarios where SUs report over fading channels, and where the inhomogeneity of the physical environment results in some SUs having stronger reporting channels than others. 


\subsection{Scope and Contributions}

This thesis considers SUs that sense a single PU using energy detection. The Rayleigh fading sensing channels are modelled as independent and non-identical. We also assume that we have limited reporting bandwidth. Thus, after sensing, each SU makes a local decision ("active" or "idle") about the PU that is reported to the base station over a Rayleigh fading channel. All reporting channels are also modelled as independent and non-identical. The base station acts as a FC by inferring the decision of each SU before combining the decisions with an $M$-out-of- $K$ rule to reach a global decision. $M$-out-of- $K$ rules facilitate closed-form expressions for the network false alarm and missed detection probabilities.

The contributions of this thesis are as follows:

1. In constrast to existing work, we allow the SUs to increase the number of reporting time slots by sacrificing sensing time slots. This is motivated by [10], which showed notable performance gain by perfectly reporting 2 bits instead of 1. However, in contrast to [10], our design repeats the binary decisions of the SUs to increase the Signal-to-Noise Ratios (SNRs) of the faded and noisy reporting channels.

2. We derive expressions for the false alarm and missed detection probabilities of the network, as functions of the SU decision thresholds and the number of reporting time slots for each SU. Our analysis assumes that each SU senses long enough such that its energy detection decision variables can be modelled as Gaussian distributed via the central limit theorem. We derive these probabilities for instantaneous reporting channel realizations as well as for average reporting channels. We begin with the single-SU network case as a foundation to extend to the general, multi-SU network case.

3. We apply upper bounds and impose convex constraints that make the false alarm and missed detection probabilities jointly convex with respect to the SU decision thresholds and the number of reporting time slots for each SU. This enables the application of convex optimization techniques to quickly and efficiently minimize the secondary network's false alarm probability under a target missed detection probability (or vice versa). We consider the 
two cases where the instantaneous and average reporting channel gains are known for optimization to investigate the tradeoffs of optimizing less frequently.

4. Simulation results show that our proposed upper bounds are reasonably tight while enabling very good performance with only a small number of sensors. We also show that the secondary network sensing performance benefits substantially from the ability to optimize the number of reporting time slots over optimization of the local decision thresholds alone.

\subsection{Organization}

The rest of this thesis is organized as follows. In Chapter 2, we introduce the cognitive radio network model with the notation used for the rest of the work. In Chapter 3, we derive the sensing error probabilities of the single-SU network and formulate convex upper bounds. In Chapter 4, we extend the work in Chapter 3 to the general, multi-SU network. In Chapter 5, we present and discuss detailed simulation results for a secondary network in both the single-SU and multi-SU cases. In Chapter 6, we present conclusions and possibilities for future work. 


\section{Chapter 2}

\section{Network Model}

This chapter introduces the network model studied and the notation used throughout the rest of this thesis.

In Section 2.1, we describe the topology of the primary and secondary networks. We present our assumptions regarding the operation of the two networks, and we briefly motivate the need for spectrum sensing in this environment.

In Sections 2.2 and 2.3, we describe the secondary network's sensing and reporting channels, respectively. We also define how much the secondary network knows about these channels.

In Section 2.4, we formally define the secondary network's probabilities of false alarm and missed detection, which are the core performance metrics for our spectrum sensing analysis. We include notation for referring to specific subsets of SUs in the network.

\subsection{Network Topology}

There is a single transmitting PU, as in Fig. 2.1. The PU could be serving, for example, as the broadcast tower for a licensed network. The PU transmits with average transmission power $P_{t}$ over the frequency band monitored by the secondary network. For convenience of the analysis, we do not consider the broadcasting carrier frequency by representing all signals with their complex baseband equivalents.

The secondary network has $K$ SUs in addition to its own base station. The 


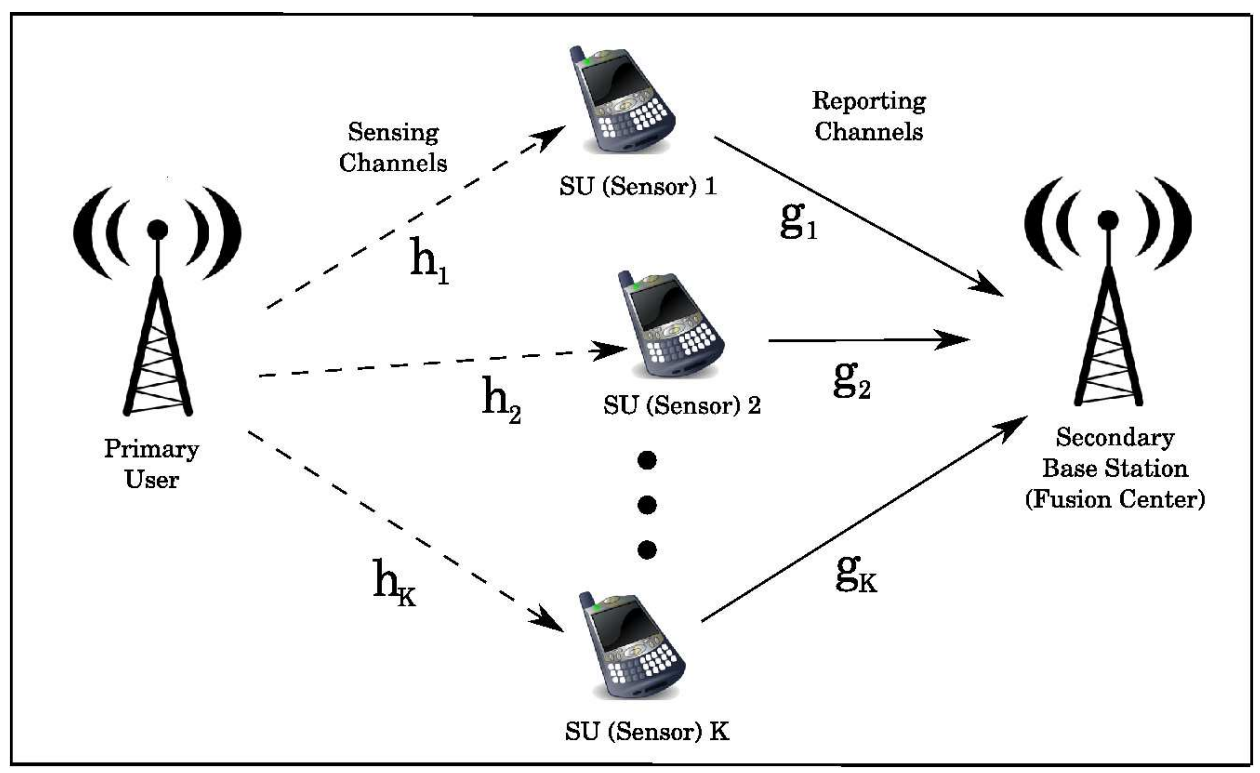

Figure 2.1: Secondary network near a primary transmitter.

network intends to utilize the PU's frequency band only when it believes that the PU is idle. Thus, we do not need to consider the PU's intended receivers in our analysis. We assume that the secondary network is not in direct communication with the PU, so the secondary network must perform spectrum sensing to assess the current activity of the PU. The spectrum sensing is cooperative in the sense that the SUs make local sensing decisions that are reported to the base station, which fuses the results to obtain a global sensing decision. Thus, we hereafter refer refer to the SUs as (local) sensors and the base station as the Fusion Center (FC).

\subsection{Sensing Channels}

The channel between the PU and the $k$ th sensor, $h_{k}$, is Rayleigh fading with variance $\sigma_{h, k}^{2}, k \in\{1,2, \ldots, K\}$. The PU-SU channels are modelled as independent and non-identically distributed. The signal received by the $k$ th sensor is impaired by complex Additive White Gaussian Noise (AWGN) with variance $\sigma_{n, k}^{2}$. Thus, the $k$ th sensor's sensing SNR is $\bar{\gamma}_{S, k}=P_{t} \sigma_{h, k}^{2} / \sigma_{n, k}^{2}$, and we let $\bar{\gamma}_{S}=\left[\bar{\gamma}_{S, 1}, \bar{\gamma}_{S, 2}, \ldots, \bar{\gamma}_{S, K}\right]$.

Each sensor has a fixed interval of $N$ time slots during which it has to perform 


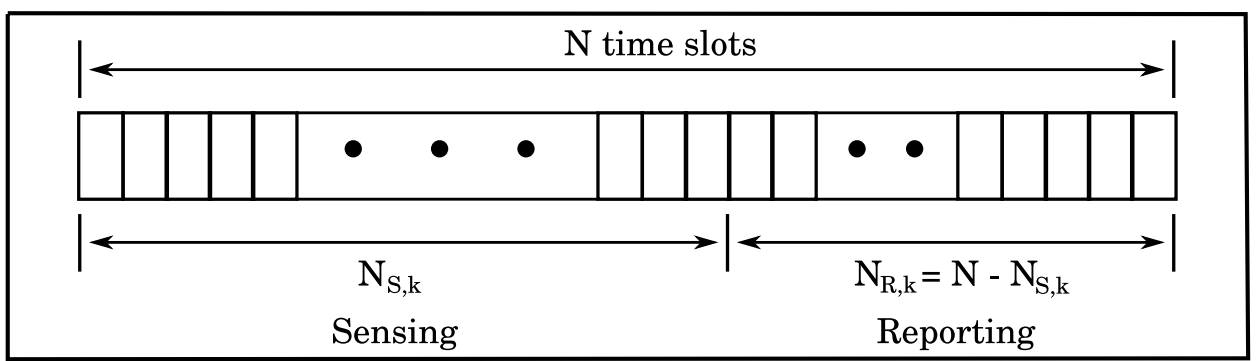

Figure 2.2: Division of sensing and reporting time slots for the $k$ th sensor.

sensing and reporting with a single antenna, as in Fig. 2.2. The time slots are assumed to all be of the same length, whether for sensing or reporting. The sensing channel is assumed to have a coherence time sufficiently long for $h_{k}$ to be assumed constant for the entire interval. We also assume that the PU is either active or idle for the entire interval, i.e., it does not cease or resume transmission within the interval. This is not a particularly strong assumption; [19] shows only a minor improvement for energy detectors that take into account PUs that arrive or depart randomly. Furthermore, we assume that each sensor has an accurate estimate of $P_{t} \sigma_{h, k}^{2}$, but does not know the instantaneous fading gain $h_{k}$. Therefore, the sensors use energy detection by sensing for $N_{S, k}$ time slots and applying decision threshold $\tau_{k}$ to decide whether the PU is active or idle.

\subsection{Reporting Channels}

The remaining $N_{R, k}=N-N_{S, k}$ time slots are used by the local sensor to report its binary decision to the Fusion Center (FC). Each sensor transmits +1 if the PU is deemed active, and -1 otherwise. The reporting channel between the $k$ th sensor and the FC, $g_{k}$, is Rayleigh fading with variance $\sigma_{g, k}^{2}$. The channels between the SUs and the FC are modelled as orthogonal, independent, and non-identically distributed. The instantaneous reporting channel gains and variances are assumed to be known at the fusion center.

The repeated reports are impaired by complex AWGN with variances $\sigma_{z, k}^{2}$. Thus, the $k$ th sensor's instantaneous reporting SNR is $\gamma_{R, k}=\left|g_{k}\right|^{2} / \sigma_{z, k}^{2}$, and we let $\gamma_{\mathbf{R}}=\left[\gamma_{R, 1}, \gamma_{R, 2}, \ldots, \gamma_{R, K}\right]$. The $k$ th sensor's average reporting SNR is $\bar{\gamma}_{R, k}=$ 
$\sigma_{g, k}^{2} / \sigma_{z, k}^{2}$, and we let $\bar{\gamma}_{\mathbf{R}}=\left[\bar{\gamma}_{R, 1}, \bar{\gamma}_{R, 2}, \ldots, \bar{\gamma}_{R, K}\right]$. We assume that $\gamma_{\mathbf{R}}$ is constant for the interval of $N$ time slots, while $\bar{\gamma}_{\mathbf{S}}$ and $\bar{\gamma}_{\mathbf{R}}$ are constant for multiple intervals.

\subsection{Sensing Performance Metrics}

Our goal is to detect when the PU is idle, so we are interested in characterizing the false alarm and missed detection probabilities. Let $H$ define the current state of the PU, where $H=1$ means that the PU is active and $H=0$ means that it is idle. The FC makes global decision $\hat{H}$, defined analogously to $H$. The network false alarm probability $P_{f F C}$ and missed detection probability $P_{m F C}$ are defined as

$$
\begin{gathered}
P_{f F C}=\operatorname{Pr}\{\hat{H}=1 \mid H=0\}, \\
P_{m F C}=\operatorname{Pr}\{\hat{H}=0 \mid H=1\} .
\end{gathered}
$$

If the $\mathrm{FC}$ is basing its decision on only the inferred decision from the $k$ th sensor, using the instantaneous reporting channel realization of that sensor, then we define these single-sensor probabilities as $P_{m F C, k}$ and $P_{f F C, k}$.

For fusion rule analysis, we must be able to refer to subsets of the sensors in the network. Let $\mathbf{S}_{K, i}$ be the union of all sets that combine $i$ out of a total of $K$ sensors, i.e., $\mathbf{S}_{K, i}$ contains $\left(\begin{array}{c}K \\ i\end{array}\right)$ sets of $i$ sensors. Furthermore, let $S_{K, i, j}$ be the $j$ th set in $\mathbf{S}_{K, i}$. The ordering of these sets is arbitrary but constant. 


\section{Chapter 3}

\section{Single-Sensor Network}

In this chapter, we derive and analyze $P_{m F C, k}$ and $P_{f F C, k}$ at the fusion center for a given reporting channel. We present a set of constraints that enable upper bounds on $P_{m F C, k}$ and $P_{f F C, k}$ to be convex with respect to the sensor's local decision threshold and the number of reporting time slots. $P_{m F C, k}$ and $P_{f F C, k}$ are also averaged over the Rayleigh-faded reporting channel (then written as $\bar{P}_{m F C, k}$ and $\bar{P}_{f F C, k}$, respectively), for which the same constraints ensure convexity. The bounds enable us to optimize $P_{m F C, k}$ and $P_{f F C, k}$, and in Chapter 4 they facilitate the relaxation of the general $K$-sensor problem to a convex optimization problem. Detailed proofs of some of the results in this chapter are omitted for narrative clarity and are deferred to the appendices.

In Section 3.1, we derive the analytical forms of $P_{m F C, k}$ and $P_{f F C, k}$, assuming that the sensor's sensing decision variables can be modelled as Gaussian distributed. We also assume that the FC uses a coherent detector for receiving the sensor's reporting signal. The sensor is able to sacrifice sensing time slots for more reporting time slots in order to increase the reporting SNR.

In Section 3.2, we derive closed-form upper bounds on $P_{m F C, k}$ and $P_{f F C, k}$. These bounds are shown to be jointly convex with respect to the local sensor decision threshold and the number of reporting time slots with the imposition of a set of convex constraints.

In Section 3.3, we derive $\bar{P}_{m F C, k}$ and $\bar{P}_{f F C, k}$ by averaging $P_{m F C, k}$ and $P_{f F C, k}$ over the Rayleigh-faded reporting channel, respectively, while still assuming coherent 
detection at the FC. The intent is to save on computation and communication time by optimizing less often, i.e., only when the variance of the reporting channel changes. The constraints imposed in Section 3.2 still apply here for convexity.

In Section 3.4, we formulate the single-sensor optimization problem where we minimize the false alarm probability while satisying a target for the missed detection probability.

\subsection{FC Performance}

\subsubsection{Local Sensing}

For our analysis, as in [18], we assume that $N_{S, k}$ is large enough such that the energy detection decision variables at the local sensor can be modelled as Gaussian distributed via the central limit theorem. We favor the Gaussian approximation approach to facilitate tractability, unlike the analysis in [7], which relies on the moment generating functions of the received signals. Thus, if the PU is idle, then the variance of the signal received at the sensor is $\sigma_{n, k}^{2} / N_{S, k}$, and we obtain the probability of false alarm at the local sensor, $P_{f L, k}$, as [18, Eq. 16]

$$
P_{f L, k}=Q\left(\left(\tau_{k} / \sigma_{n, k}^{2}-1\right) \sqrt{N-N_{R, k}}\right),
$$

where $Q(\cdot)$ is the Gaussian $Q$-function. We immediately see that if $\tau_{k}<\sigma_{n, k}^{2}$, then the argument of $Q(\cdot)$ would be negative and $P_{f L, k}>0.5$. Furthermore, an active PU would only add to the energy of the signal received at the local sensor. Thus, we will subsequently assume that $\tau_{k} \geq \sigma_{n, k}^{2}$, and the corresponding expression for the probability of missed detection at the local sensor, $P_{m L, k}$, averaged over the Rayleigh-faded sensing channel, is [18, Eq. 26]

$$
P_{m L, k}=1-\exp \left(-\xi /\left(\overline{P_{t}} \sigma_{h, k}^{2}\right)\right)+I,
$$


where

$$
\begin{aligned}
I & =\frac{1}{\sqrt{2 \pi} \overline{P_{t}} \sigma_{h, k}^{2}} \exp \left(\frac{-\xi}{\overline{P_{t}} \sigma_{h, k}^{2}}\right) \int_{0}^{\frac{\pi}{2}} \sin \theta \exp \left(\frac{\sin ^{2} \theta}{2 \bar{P}_{t}^{2} \sigma_{h, k}^{4}}\right) S d \theta, \\
S & =\left[1+\operatorname{erf}\left(\frac{\sin \theta}{\sqrt{2} \overline{P_{t}} \sigma_{h, k}^{2}}-\frac{\xi}{\sqrt{2} \sin \theta}\right)-2 \operatorname{erf}\left(\frac{\sin \theta}{\sqrt{2} \overline{P_{t}} \sigma_{h, k}^{2}}\right)\right], \\
\xi & =\left(\tau_{k} / \sigma_{n, k}^{2}-1\right) \sqrt{N_{S, k}}, \\
\bar{P}_{t} & =P_{t} \sqrt{N_{S, k}} / \sigma_{n, k}^{2},
\end{aligned}
$$

and $\operatorname{erf}(\cdot)$ is the error function [20, p. 406]. Eqs. (3.1) and (3.2) (ignoring $I$ in the latter) show that increasing $\tau_{k}$ causes an increase in $P_{m L, k}$ and a decrease in $P_{f L, k}$, and vice versa.

\subsubsection{Sensor Reporting}

We next derive an expression for $P_{m F C, k}$. The sensor makes 1-bit decision $\hat{H}_{L, k}$ and transmits it to the FC with a repetition code as

$$
b_{k}[n]=b_{k}=\left\{\begin{array}{lll}
+1 & \text { if } \hat{H}_{L, k}=1, & N_{S, k}+1 \leq n \leq N \\
-1 & \text { if } \hat{H}_{L, k}=0, & N_{S, k}+1 \leq n \leq N
\end{array}\right.
$$

We note that, for a single reporting interval, if $\hat{H}_{L, k}=1$, then $b_{k}=-1$ with probability $P_{m L, k}$ and $b_{k}=+1$ with probability $\left(1-P_{m L, k}\right)$. The FC receives

$$
y_{k}[n]=g_{k} b_{k}[n]+z_{k}[n], \quad N_{S, k}+1 \leq n \leq N,
$$

where $z_{k}[n]$ is complex AWGN. The fusion center forms

$$
y_{k}=\frac{1}{N_{R, k}} \sum_{n=N_{S, k}+1}^{N} y_{k}[n]=g_{k} b_{k}+z_{k},
$$

where $z_{k}$ is complex AWGN with variance $\sigma_{z, k}^{2} / N_{R, k}$. Thus, we see how increasing the number of reporting time slots corresponds to an increase in the effective reporting SNR. We construct a coherent detector where we multiply $y_{k}$ by $g_{k}^{*} /\left|g_{k}\right|$ and take the real component $\left((\cdot)^{*}\right.$ denotes complex conjugation). Thus, we obtain 
$P_{m F C, k}$ as

$$
\begin{aligned}
P_{m F C, k} & =\operatorname{Pr}\left\{\left|g_{k}\right| b_{k}+\Re\left\{g_{k}^{*} z_{k} /\left|g_{k}\right|\right\}<0 \mid H=1\right\} \\
& =\operatorname{Pr}\left\{\Re\left\{g_{k}^{*} z_{k} /\left|g_{k}\right|\right\}<\left|g_{k}\right|\right\}\left(1-P_{m L, k}\right)+\operatorname{Pr}\left\{\Re\left\{g_{k}^{*} z_{k} /\left|g_{k}\right|\right\}<-\left|g_{k}\right|\right\} P_{m L, k} \\
& =Q\left(\left|g_{k}\right| \sqrt{2 N_{R, k} / \sigma_{z, k}^{2}}\right)\left(1-P_{m L, k}\right)+Q\left(-\left|g_{k}\right| \sqrt{2 N_{R, k} / \sigma_{z, k}^{2}}\right) P_{m L, k},
\end{aligned}
$$

where $\mathfrak{R}\{\cdot\}$ denotes the real component of a complex number. From the first line of (3.10), we see that the FC uses 0 as a decision threshold. Given that one of our two design parameters is the local sensor decision threshold $\tau_{k}$, one may validly question whether the network could benefit by making the FC threshold variable. In fact, this threshold was initially incorporated as a variable in our analysis. However, it was found to complicate the convexity conditions while providing negligible performance improvement. Thus, we set it to 0 .

Analogously, $P_{f F C, k}$ is obtained as

$$
P_{f F C, k}=Q\left(-\left|g_{k}\right| \sqrt{2 N_{R, k} / \sigma_{z, k}^{2}}\right) P_{f L, k}+Q\left(\left|g_{k}\right| \sqrt{2 N_{R, k} / \sigma_{z, k}^{2}}\right)\left(1-P_{f L, k}\right)
$$

\subsection{Closed-Form, Convex Bounds on Performance}

\subsubsection{Upper Bounds on Performance}

Unfortunately, we cannot obtain (3.10) in closed form due to the integral in (3.3). We will, however, require a closed-form expression in order to define tractable optimization problems. Therefore, we derive a closed-form upper bound on (3.3) that leads to a closed-form upper bound on (3.10).

Theorem 1 (Upper bound on (3.3)): Eq. (3.3) can be upper-bounded in closed 
form by

$$
\begin{aligned}
I_{\text {bound }}= & A_{k} x_{k}\left[B_{k}-A_{k} \frac{N_{S_{\max }}-\left(N-N_{R, k}\right)}{\sqrt{2 \pi} N_{S_{\max }^{3 / 2}}^{3}}\right]+C_{k}\left(2 \exp \left(-A_{k} x_{k}\right)+\exp \left(-N_{S_{\min }} x_{k}^{2}\right)\right) \\
& +\frac{D_{k}}{\sqrt{\left(N-N_{R, k}\right)}} \exp \left(-A_{k} x_{k}\right) \\
& +\frac{A_{k}}{\sqrt{2 \pi}} \frac{\sigma_{n, k}^{2}}{N_{S_{\min }} x_{k}-A_{k}+\sqrt{N_{S_{\min }}}} \exp \left(\frac{-A_{k} x_{k}-N_{S_{\min }} x_{k}^{2}}{2}\right),
\end{aligned}
$$

where

$$
x_{k}=\tau_{k} / \sigma_{n, k}^{2}-1,
$$

positive constants $A_{k}, B_{k}, C_{k}$, and $D_{k}$ are given by

$$
\begin{aligned}
& A_{k}=\frac{\sigma_{n, k}^{2}}{P_{t} \sigma_{h, k}^{2}}, \\
& B_{k}=\exp \left(\frac{1}{2 N_{S_{\max }}} A_{k}^{2}\right) \operatorname{erf}\left(\frac{1}{\sqrt{2 N_{S_{\max }}}} A_{k}\right) \text {, } \\
& C_{k}=\frac{1}{25} \operatorname{erfi}\left(\frac{1}{\sqrt{2 N_{S_{\min }}}} A_{k}\right) \exp \left(-\frac{1}{2 N_{S_{\max }}} A_{k}^{2}\right) \text {, } \\
& D_{k}=\frac{1}{\sqrt{2 \pi}} A_{k}\left(2+\frac{\pi}{2}\left(\frac{\sqrt{N_{S_{\max }}} P_{t} \sigma_{h, k}^{2}}{\sigma_{n}^{2}+\sqrt{N_{S_{\max }}} P_{t} \sigma_{h, k}^{2}}-1\right)\right) \text {, }
\end{aligned}
$$

and erfi(.) is the imaginary error function [20, p. 427]. $N_{S_{\min }}$ and $N_{S_{\max }}$ are bounds on $N_{S, k}$ that are introduced to simplify convexity analysis with minimal impact on the value of $I_{\text {bound }}$.

Proof: Refer to Appendix A. The proof converts the error functions in (3.3) into equivalent $Q$-functions, and then applies the "supertight bound" on the $Q$-function given in [21] in addition to two Taylor series approximations.

If the argument of $Q(\cdot)$ is negative, then $0.5<Q(\cdot)<1$. We also aim to achieve low $P_{m L, k}$ (i.e., $P_{m L, k} \ll 1$ ). Therefore, we upper-bound (3.10) by

$$
P_{m F C, k} \leq Q\left(\left|g_{k}\right| \sqrt{2 N_{R, k} / \sigma_{z, k}^{2}}\right)+A_{k} x_{k}+I_{\text {bound }},
$$


where we used a first degree Taylor series approximation of the non-integral components of $P_{m L, k}$, i.e., $1-\exp \left(-\xi /\left(\bar{P}_{t} \sigma_{h, k}^{2}\right)\right)$, at $\tau_{k}=\sigma_{n, k}^{2}$, as otherwise $P_{m L, k}$ (ignoring the $I$ term) is concave with respect to $\tau_{k}$.

Eq. (3.11) is already in closed form. However, to define tractable optimization problems, we upper-bound it by

$$
P_{f F C, k} \leq Q\left(\left|g_{k}\right| \sqrt{2 N_{R, k} / \sigma_{z, k}^{2}}\right)+Q\left(x_{k} \sqrt{N-N_{R, k}}\right),
$$

where we used again that $0.5<Q(\cdot)<1$ when the argument of $Q(\cdot)$ is negative, and $P_{f L, k} \ll 1$.

\subsubsection{Convexity of Upper Bounds}

We relax $N_{R, k}$ to be a real number for optimzation (though in simulations and in practice we round it to the nearest natural number), and present the following theorem:

Theorem 2 (Convexity of (3.15) and (3.16)): Eqs. (3.15) and (3.16) can be shown to be jointly convex with respect to $\tau_{k}$ and $N_{R, k}$, if we impose the following convex constraints:

$$
\begin{array}{r}
\sigma_{n, k}^{2}-\tau_{k} \leq 0, \\
1 \leq N_{R, k} \leq N_{R_{\max }}, \\
x_{k}^{-2}-(1+\sqrt{2}) N_{S_{\min }} \leq 0, \\
\frac{\sigma_{n, k}^{2}}{N-N_{R, k}}-A_{k} x_{k}<0, \\
2 A_{k} x_{k}-E \leq 0, \\
A_{k}^{2} \exp \left(2 A_{k} x_{k}\right)+\frac{A_{k}^{2}\left(N-N_{R, k}\right)^{3}}{N_{S_{\max }^{3}}} \exp (E)-\frac{2 A_{k} D_{k} N_{S_{\min }}^{3 / 2}}{N_{S_{\max }^{3 / 2}}}-D_{k}^{2} \leq 0,
\end{array}
$$

where $N_{R_{\max }}=N-N_{S_{\min }}, x_{k}$ is as in (3.13), and $E$ is a tunable parameter, defined in (3.21), that limits the maximum value of $\tau_{k}$.

Proof: We prove the convexity of (3.16) and (3.15) in Appendices B and C, respectively, by showing that the Hessians of (3.15) and (3.16) are non-negative once 
(3.17) to (3.22) are imposed [22, 23]. As discussed earlier, (3.17) is imposed so that we are able to use (3.2). The local sensor must be able to report for at least one time slot, so $N_{R, k} \geq 1$. Eq. (3.19) couples $\tau_{k}$ with $N_{R, k}$ to make $I_{\text {bound }}$ convex with respect to $\tau_{k}$. Eq. (3.20) is used with the "supertight bound" [21] to evaluate (3.3) in closed form. Eq. (3.22) is less intuitive, but it ensures the joint convexity of $\tau_{k}$ and $N_{R, k}$ in $I_{\text {bound }}$. Eq. (3.21) is imposed to improve the tightness of (3.22) while maintaining convexity.

We observe that there are both upper and lower bounds on $\tau_{k}$ and $N_{R, k}$. This means that there are both upper and lower limits on the values of $P_{m F C, k}$ and $P_{f F C, k}$, i.e., we cannot achieve arbitrarily small or large $P_{m F C, k}$ or $P_{f F C, k}$ while maintaining convexity. This phenomenon will become evident when analyzing Receiver Operating Characteristics (ROCs).

\subsection{Performance Averaged Over Reporting Channels}

The sensing error performance as given by (3.15) and (3.16) must be re-evaluated every time the reporting channel changes. Thus, the coherence time of the reporting channel influences the optimization frequency. Significant network resources (both time and energy) could be consumed performing computations and communicating both environment parameters and optimal results. It may then be beneficial to optimize less frequently, which we may do if we average performance over the Rayleigh-faded reporting channel.

Eqs. (3.15) and (3.16) share a common reporting component that we define as

$$
P_{R, k}=Q\left(\left|g_{k}\right| \sqrt{2 N_{R, k} / \sigma_{z, k}^{2}}\right) \text {. }
$$

We can save on computation time and the corresponding communications overhead by averaging (3.23) over the Rayleigh-faded reporting channel, since $\bar{\gamma}_{R, k}$ is constant for longer than $\gamma_{R, k}$. This probability, $\bar{P}_{R, k}$, is defined and upper-bounded in the following theorem: 
Theorem 3 (Upper bound on $\bar{P}_{R, k}$ ): $\bar{P}_{R, k}$ is defined and then upper-bounded by

$$
\bar{P}_{R, k}=\frac{\sigma_{z, k}^{2}}{\pi N_{R, k} \sigma_{g, k}^{2}} \int_{0}^{\frac{\pi}{2}} \frac{d \theta}{\frac{\sigma_{z, k}^{2}}{N_{R, k} \sigma_{g, k}^{2}}+1 / \sin ^{2} \theta} \leq \frac{\sigma_{z, k}^{2}}{4 N_{R, k} \sigma_{g, k}^{2}} .
$$

Proof: Refer to Appendix D The proof of the definition is found using the Craig representation of the $Q$-function (as in [18]) and a variable substitution. The upper bound is derived by assuming that $\sigma_{z, k}^{2} /\left(N_{R, k} \sigma_{g, k}^{2}\right)$ is relatively small (though we are not required to impose another constraint).

The exact form in (3.24) allows us to average (3.10) and (3.11) over $g_{k}$ and write

$$
\begin{aligned}
& \bar{P}_{m F C, k}=\bar{P}_{R, k}\left(1-P_{m L, k}\right)+\left(1-\bar{P}_{R, k}\right) P_{m L, k}, \\
& \bar{P}_{f F C, k}=\bar{P}_{R, k}\left(1-P_{f L, k}\right)+\left(1-\bar{P}_{R, k}\right) P_{f L, k} .
\end{aligned}
$$

The upper bound in (3.24) is trivially convex with respect to $N_{R, k}$. Eqs. (3.15) and (3.16) become

$$
\begin{aligned}
& \bar{P}_{m F C, k} \leq A_{k} x_{k}+I_{\text {bound }}+\frac{\sigma_{z, k}^{2}}{4 N_{R, k} \sigma_{g, k}^{2}}, \\
& \bar{P}_{f F C, k} \leq Q\left(x_{k} \sqrt{N-N_{R, k}}\right)+\frac{\sigma_{z, k}^{2}}{4 N_{R, k} \sigma_{g, k}^{2}}
\end{aligned}
$$

respectively. Note that, since (3.17) to (3.22) are derived from the sensing component of (3.15) and (3.16), (3.27) and (3.28) are jointly convex with respect to $\tau_{k}$ and $N_{R, k}$ under the same set of constraints.

\subsection{Single-Sensor Optimization Problem}

Our goal is to optimize performance by minimizing one probability while satisfying a target for the other probability. Since we would like to guarantee a ceiling on interference to the $\mathrm{PU}$, we choose a target missed detection probability, $P_{m F C_{T A R}}$, as in [13]. Thus, when the instantaneous reporting channel is known, the optimization 
problem for the single-sensor network can be formulated as

$$
\begin{array}{cl}
\text { minimize } & P_{f F C, k} \\
\text { subject to } & P_{m F C, k} \leq P_{m F C_{T A R}} \\
& (3.17) \text { to }(3.22) .
\end{array}
$$

Analogously, when only the average reporting channel is known, the optimization problem for the single-sensor network can be formulated as

$$
\begin{array}{ll}
\text { minimize } & \bar{P}_{f F C, k} \\
\text { subject to } & \bar{P}_{m F C, k} \leq P_{m F C_{T A R}} \\
& (3.17) \text { to }(3.22) .
\end{array}
$$

Due to the convexity of the objective function and all of the constraints, problems (3.29) and (3.30) can both be solved by efficient algorithms such as the interior-point method [22].

We emphasize that the frequency for solving (3.29) depends primarily on the reporting channel coherence time (though also on the sensing channel coherence time). Solving (3.29) offers improved spectrum sensing performance while using (3.30) is less demanding on network resources. 


\section{Chapter 4}

\section{Multi-Sensor Network}

In this chapter, we derive and analyze $P_{m F C}$ and $P_{f F C}$ for the $K$-sensor network using $P_{m F C, k}$ and $P_{f F C, k}$. We can analogously derive $\bar{P}_{m F C}$ and $\bar{P}_{f F C}$ (averaging probabilities over reporting channels) using $\bar{P}_{m F C, k}$ and $\bar{P}_{f F C, k}$ if only the average reporting channels are known. We upper-bound $P_{m F C}$ and $P_{f F C}$ to obtain a generalized convex multiplicative problem [24] for OR-rule. Subsequently, we relax this problem to arrive at a convex optimization problem and extend the analysis to any $M$-out-of- $K$ rule. Detailed proofs of some of the results in this chapter are omitted for narrative clarity and are deferred to the appendices. Throughout this chapter we assume that $K \geq 2$.

In Section 4.1, we present the analytical forms of $P_{m F C}$ and $P_{f F C}$ as functions of the individual $P_{m F C, k}$ and $P_{f F C, k}$. These expressions apply to any $M$-out-of- $K$ rule. We derive upper bounds on both $P_{m F C}$ and $P_{f F C}$. For OR-rule, we show that the upper bounds lead to a generalized convex multiplicative problem for network optimization.

In Section 4.2, we relax the optimization problem for OR-rule to arrive at a set of independent convex optimization subproblems. We achieve this by uncoupling the missed detection target probability, resulting in one convex optimization subproblem corresponding to each sensor. We briefly discuss the tradeoffs of solving the problems at the $\mathrm{FC}$ versus at the sensors.

In Section 4.3, we show that the approach used to formulate a set of convex optimization subproblems when using OR-rule can be generalized to any $M$-out- 
of- $K$ rule.

\subsection{Optimization Problem}

The fusion center combines the $K$ inferred local sensor decisions into a global decision using an $M$-out-of- $K$ rule. We focus attention on analysis for OR-rule (i.e., $M=1$ ) for three reasons: 1) simplicity, 2) the analysis will lead to optimization of any $M$-out-of- $K$ rule as a convex problem, and 3) we will suggest in Section 5.1 that optimized network performance will favor a rule that multiplies missed detection probabilities, i.e., OR-rule.

\subsubsection{Exact Expressions for Decision Fusion}

Conveniently, $P_{m F C}$ and $P_{f F C}$ can be writen as functions of the corresponding single-sensor probabilities. $P_{f F C}$ for any $M$-out-of- $K$ rule, using our notation, is [25, Eq. 10]

$$
P_{f F C}=\sum_{i=M}^{K}\left\{\left(\sum_{p=0}^{i-M}(-1)^{p}\left(\begin{array}{c}
i \\
p
\end{array}\right)\right)\left(\sum_{S_{K, i, j} \in \mathbf{S}_{K, i}} \prod_{k \in S_{K, i, j}} P_{f F C, k}\right)\right\} .
$$

In other words, we sum every combination of products of $M$ false alarm probabilities, $M+1$ false alarm probabilities, ..., and $K$ false alarm probabilities, and every combination has a coefficient calculated by [26, p. 165]

$$
\sum_{p=0}^{i-M}(-1)^{p}\left(\begin{array}{c}
i \\
p
\end{array}\right)=(-1)^{i-M}\left(\begin{array}{c}
i-1 \\
i-M
\end{array}\right)
$$

Similarly, [25] provides an expression for the probability of detection. However, $P_{m F C}$ is more relevant here, which we write as

$$
P_{m F C}=\sum_{i=K-M+1}^{K}\left\{\left(\sum_{p=0}^{i+M-K-1}(-1)^{p}\left(\begin{array}{c}
i \\
p
\end{array}\right)\right)\left(\sum_{S_{K, i, j} \in \mathbf{S}_{K, i}} \prod_{k \in S_{K, i, j}} P_{m F C, k}\right)\right\},
$$


where [26, p. 165]

$$
\sum_{p=0}^{i+M-K-1}(-1)^{p}\left(\begin{array}{l}
i \\
p
\end{array}\right)=(-1)^{i+M-K-1}\left(\begin{array}{c}
i-1 \\
i+M-K-1
\end{array}\right)
$$

\subsubsection{Exact Expressions and Bounds for Decision Fusion}

Our goal is to upper bound $P_{f F C}$ and $P_{m F C}$ by only considering the products of the fewest probabilities, i.e. by only considering $i=M$ in (4.1) and $i=K-M+1$ in (4.3). Products of more probabilities should be smaller since they are the product of more positive terms less than or equal to one. However, this may not be the case if the coefficients become large. We impose a constraint on the average sensing error probability that guarantees an upper bound, as given in the following theorem:

Theorem 4 (Upper Bound on $P_{f F C}$ and $P_{m F C}$ ): $P_{f F C}$ and $P_{m F C}$ are upper-bounded by

$$
\begin{aligned}
& P_{f F C} \leq \sum_{S_{K, M, j} \in \mathbf{S}_{K, M}} \prod_{k \in S_{K, M, j}} P_{f F C, k}, \\
& P_{m F C} \leq \sum_{S_{K, K-M+1, j} \in \mathbf{S}_{K, K-M+1}} \prod_{k \in S_{K, K-M+1, j}} P_{m F C, k},
\end{aligned}
$$

if we impose the following constraints:

$$
\begin{aligned}
& \frac{\sum_{k=1}^{K} P_{f F C, k}}{K} \leq \frac{2 M+4}{K M+K-M^{2}-2 M-1} \\
& \frac{\sum_{k=1}^{K} P_{m F C, k}}{K} \leq \frac{2 K-2 M+6}{K M-2 K-M^{2}+4 M-4}
\end{aligned}
$$

which are convex with respect to $\tau_{k}$ and $N_{R, k}, \forall k$.

Proof: Refer to Appendix E. We compare binomial coefficients to show that, when applying (4.7) and (4.8), the negative terms in (4.2) and (4.3) are greater than the positive terms in (4.2) and (4.3) that are ignored in (4.5) and (4.6). 


\subsubsection{Bounds Applied to OR-Rule}

For OR-rule $(M=1)$, the upper bound (4.6) for $P_{m F C}$ is equivalent to (4.3), so we can ignore (4.8). The upper bound on $P_{f F C}$ is

$$
P_{f F C} \leq \sum_{k=1}^{K} P_{f F C, k}
$$

which is tight when the individual $P_{f F C, k}$ terms are small. The upper bound (4.9) is convex since it is a sum of convex functions. Furthermore, (4.7) becomes

$$
\sum_{k=1}^{K} P_{f F C, k} \leq \frac{6 K}{2 K-4}
$$

Assuming physically reasonable $P_{f F C}$ (i.e., $\left.P_{f F C, k}<1\right)$, (4.10) can also be ignored. Our optimization problem becomes

$$
\begin{array}{ll}
\text { minimize } & \sum_{k=1}^{K} P_{f F C, k} \\
\text { subject to } & \prod_{k=1}^{K} P_{m F C, k} \leq P_{m F C_{T A R}} \\
& (3.17) \text { to }(3.22), \forall k,
\end{array}
$$

where we use upper bounds for $P_{m F C, k}$ and $P_{f F C, k}$ (from (3.15) and (3.16)).

\subsubsection{Generalized Convex Multiplicative Programming}

Since $P_{m F C}$ is the product of convex functions, (4.11) is not convex in general. Problem (4.11) is an example of generalized convex multiplicative programming, where a problem that is otherwise convex has either an objective term or one constraint that is a product of convex functions. This is a relatively new field of optimization [24], though it has been shown that such a problem can be transformed into a series of convex problems.

Problem (4.11) can be globally solved by the simplicial branch-and-reduce method described in [27]. Simplicial branch-and-reduce represents the problem in an equivalent form where $K$-simplices ( $K$-dimensional shapes with certain prop- 
erties) are used to represent the individual convex functions in the multiplicative constraint. In each iteration of the method, one of the simplices is removed and replaced with $K$ more $K$-simplices, and a corresponding convex problem is solved. With each iteration, the convex problem complexity grows from the net gain of $(K-1) K$-simplices. However, the algorithm will eventually converge to the optimal solution. In practice, the execution time of the branch-and-reduce algorithm in [27] grows prohibitively large as $K$ increases. Thus, it is of interest to find a simpler method for solving (4.11).

\subsection{Suboptimal Convex Problem for OR-Rule}

For OR-rule, we observe that $P_{m F C}$ is a product of independently convex functions, since each $P_{m F C, k}$ is only a function of its corresponding $\tau_{k}$ and $N_{R, k}$. Therefore, dividing the missed detection target in (4.11) into $K$ targets, $P_{m F C_{T A R}, k}$ (one for each sensor), will create $K$ independent convex subproblems.

A simple way to derive $P_{m F C_{T A R}, k}$ would be to take the $K$ th root of $P_{m F C_{T A R}}$, as in [12]. However, this ignores that some sensors can achieve better performance than others due to stronger sensing or reporting channels. As noted in Section 3.2.2, there is a lower limit on every achievable $P_{m F C, k}$, which will vary from sensor to sensor. Therefore, we propose scaling the target probability of each sensor based on the best achievable $P_{m F C, k}$ of that sensor. So, we first solve

$$
\begin{array}{ll}
\text { minimize } & P_{m F C, k} \\
\text { subject to } & (3.17) \text { to }(3.22),
\end{array}
$$

for all $k$ sensors, which are convex optimization problems, and define $P_{m F C_{m i n}, k}$ as the solution to (4.12). The network's minimum missed detection probability, $P_{m F C_{\text {min }}}$, using OR-rule is then

$$
P_{m F C_{\min }}=\prod_{k=1}^{K} P_{m F C_{\min }, k}
$$

Obviously, if $P_{m F C_{T A R}}=P_{m F C_{m i n}}$, then we require $P_{m F C_{T A R}, k}=P_{m F C_{m i n}, k}, \forall k$. Otherwise, at least one sensor will have an unachievable target, i.e., $P_{m F C_{T A R}, k}<$ 
$P_{m F C_{m i n}, k}$ for some $k$. When $P_{m F C_{T A R}}>P_{m F C_{m i n}}$, it is reasonable to assign relatively lower $P_{m F C_{T A R}, k}$ to sensors that have lower $P_{m F C_{m i n}, k}$. We propose achieving this by scaling each sensor's target relative to its minimum as

$$
P_{m F C_{T A R}, k}=P_{m F C_{\min }, k}\left(\frac{P_{m F C_{T A R}}}{P_{m F C_{\min }}}\right)^{1 / K}
$$

where it is then straightforward to verify that

$$
P_{m F C_{T A R}}=\prod_{k=1}^{K} P_{m F C_{T A R}, k}
$$

Thus, we relax (4.11) to arrive at the new problem

$$
\begin{array}{cl}
\text { minimize } & \sum_{k=1}^{K} P_{f F C, k} \\
\text { subject to } & P_{m F C, k} \leq P_{m F C_{T A R}, k}, \forall k \\
& (3.17) \text { to }(3.22), \forall k .
\end{array}
$$

Problem (4.16) readily decomposes into $K$ convex subproblems that can be efficiently solved [22] either at the FC or at the sensors. The FC knows $g_{k}$ and $\sigma_{z, k}^{2}, \forall k$, but in order for it to solve all subproblems, it also needs to learn $P_{t} \sigma_{h, k}^{2}$ and $\sigma_{n, k}^{2}, \forall k$, via a feedback channel. However, the $k$ th sensor knows $P_{t} \sigma_{h, k}^{2}$ and $\sigma_{n, k}^{2}$, so in order for it to solve the $k$ th subproblem it only needs to learn $\left|g_{k}\right|$ and $\sigma_{z, k}^{2}$ via a feedback channel. Either method requires feedback of the optimal $\tau_{k}$ and $N_{R, k}, \forall k$, between the sensors and the FC. For this thesis, we do not consider where (4.16) is solved.

Relaxed problem (4.16) does not in general yield the optimal solution. Recall from Section 3.2.2 that there is also an upper limit on every achievable $P_{m F C, k}$. In the event that our scaling assigns $P_{m F C_{T A R}, k}<P_{m F C_{\text {max }}, k}$ for some $k$, where $P_{m F C_{\max }, k}$ is the sensor's maximum missed detection probability, then solving (4.16) will yield $P_{m F C}<P_{m F C_{T A R}}$. There is generally a tradeoff between false alarm and missed detection, so having a missed detection probability below the target means that we are probably not minimizing the false alarm probability. Nevertheless, as will be shown in Section 5.2 of this thesis, the target scaling approach usually yields $P_{m F C}$ 
and $P_{f F C}$ that are very close to the optimal solution of (4.11).

\subsection{Suboptimal Convex Problem for Generalized Counting Rule}

We can generalize the formulation of (4.16) from (4.11) to any $M$-out-of- $K$ rule. Again, we use upper bounds for $P_{m F C, k}$ and $P_{f F C, k}$ (from (3.15) and (3.16)). We keep the same objective function as in (4.16), even though it is no longer representative of the actual $P_{f F C}$, in order to enable decomposition into convex subproblems.

$P_{m F C_{T A R}, k}$ requires scaling to achieve the upper bound defined by (4.6). As a generalization of (4.14), we propose scaling each sensor's target relative to its minimum as

$$
P_{m F C_{T A R}, k}=P_{m F C_{\text {min }}, k}\left(\frac{P_{m F C_{T A R}}}{P_{m F C_{\text {min }}}}\right)^{1 /(K-M+1)},
$$

where it is then straightforward to verify that

$$
P_{m F C_{T A R}}=\sum_{S_{K, K-M+1, j} \in \mathbf{S}_{K, K-M+1}} \prod_{k \in S_{K, K-M+1, j}} P_{m F C_{T A R}, k}
$$

As an example, we will verify (4.18) for the case of $K=3, M=2$, as follows:

$$
\begin{gathered}
\sum_{S_{K, K-M+1, j} \in \mathbf{S}_{K, K-M+1}} \prod_{k \in S_{K, K-M+1, j}} P_{m F C_{T A R}, k} \\
=P_{m F C_{T A R}, 1} P_{m F C_{T A R}, 2}+P_{m F C_{T A R}, 1} P_{m F C_{T A R}, 3}+P_{m F C_{T A R}, 2} P_{m F C_{T A R}, 3} \\
=\frac{P_{m F C_{T A R}}}{P_{m F C_{\text {min }}}}\left(P_{m F C_{\min , 1}, 1} P_{m F C_{m i n}, 2}+P_{m F C_{m i n}, 1} P_{m F C_{m i n}, 3}+P_{m F C_{m i n}, 2} P_{m F C_{m i n}, 3}\right) \\
=\frac{P_{m F C_{T A R}}}{P_{m F C_{\text {min }}}} P_{m F C_{m i n}}=P_{m F C_{T A R}}
\end{gathered}
$$

Thus, for any $M$-out-of- $K$ rule, we can solve (4.16) using $P_{m F C_{T A R}, k}, \forall k$, found by (4.17). Of course, we must also consider constraints (4.7) and (4.8), which we were able to ignore for OR-rule. 


\section{Chapter 5}

\section{Numerical Results and Discussion}

In this Chapter, we present and discuss simulation results based on the analytical results in Chapter 3 and Chapter 4.

In Section 5.1, we present Receiver Operating Characteristics (ROCs) for a single-sensor optimization problem. We show the loss in accuracy and achievable sensing performance due to bounding, and the performance loss incurred when optimizing less often (by optimizing based on the reporting channel variance instead of the instantaneous reporting channel). We show that simulations are consistent with the exact performance of the optimized network. We also consider the effects of using different interval length $N$.

In Section 5.2, we present ROCs for multi-sensor optimization problems. We compare the application of different $M$-out-of- $K$ rules, showing that OR-rule enables the best performance. We also consider the sensitivity of $P_{f F C}$ to the quality of the sensing and reporting channels using two different fusion rules while showing the benefits of optimizing the number of reporting time slots over only optimizing the local decision thresholds.

Unless otherwise noted, results in this Chapter are for a simulated secondary network with $N=5000$ time slots. We let $\sigma_{n, k}^{2}=1, \sigma_{z, k}^{2}=1$, and $P_{t}=1$ (the power units are arbitrary). For optimization, we set $N_{R_{\max }}=N-N_{S_{\min }}=1500$, $N_{S_{\max }}=N-1$, and $E=1$. We assume that the PU is transmitting a Binary PhaseShift Keying (BPSK) signal. 


\subsection{Single-Sensor Results}

Chapter 3 presented numerous bounds to formulate (3.29) as a convex optimization problem. Before considering the overall, $K$-sensor network, we consider an example to show the loss in accuracy and achievable sensing performance due to the approximations and bounds of the single-sensor network. Consider the $k$ th sensor in a simulated secondary network where, unless otherwise noted, the average sensing SNR is $\bar{\gamma}_{S, k}=-5 \mathrm{~dB}$.

Figs. 5.1 and 5.2 show the Receiver Operating Characteristic (ROC) when solving (3.29) for $\gamma_{R, k}=-6 \mathrm{~dB}$ and solving (3.30) for $\bar{\gamma}_{R, k}=-6 \mathrm{~dB}$, respectively. In Fig. 5.1, we use different interval lengths $N$ and for each we set $N_{R_{\max }}=0.3 N$ and $N_{S_{\max }}=N-1$. In Fig. 5.2, solving (3.30) once via (3.28) and (3.27) is contrasted with solving (3.29) $10^{6}$ times via (3.16) and (3.15), each time with $\gamma_{R, k}$ generated based on $\bar{\gamma}_{R, k}$, to show the loss due to optimizing less often and only using the average reporting channels instead of the instantaneous ones. Missed detection is represented by $\bar{P}_{m F C_{T A R}, k}$, defined analogously to $P_{m F C_{T A R}, k}$. In both figures, the upper bound curves were obtained from the solutions of (3.29) and (3.30). To assess bounding losses, curves are shown that were obtained using the solutions of (3.29) in the exact expressions (3.10) and (3.11) and the solutions of (3.30) in the exact expressions and (3.25) and (3.26). We also show curves obtained by using the solutions of (3.29) and (3.30) in the simulation of $10^{6}$ noise and channel realizations.

In Fig. 5.1, the looseness of the upper bound curves is primarily horizontal; the upper bounds on $P_{m F C, k}$ are looser than those on $P_{f F C, k}$ due to the upper bound on (3.3) and the Taylor series upper bound of the non-integral components of $P_{m L, k}$. The looseness means that the actual performance would be better than that given by the optimal solution of (3.29). For larger $N$, the upper bound is tighter and lower sensing error probabilities are achievable. Arbitrarily increasing $N$ is constrained by the coherence time of the sensing channel and the need of time to use the frequency band for data transmission in the event that FC decides that the PU is absent.

The smallest false alarm probability plotted for $N=1000$ in Fig. 5.1 is approximately 0.002 and constitutes the smallest possible within the convex feasibility region due to the constraints on $\tau_{k}$ and $N_{R, k}$. While it is possible to obtain a smaller 


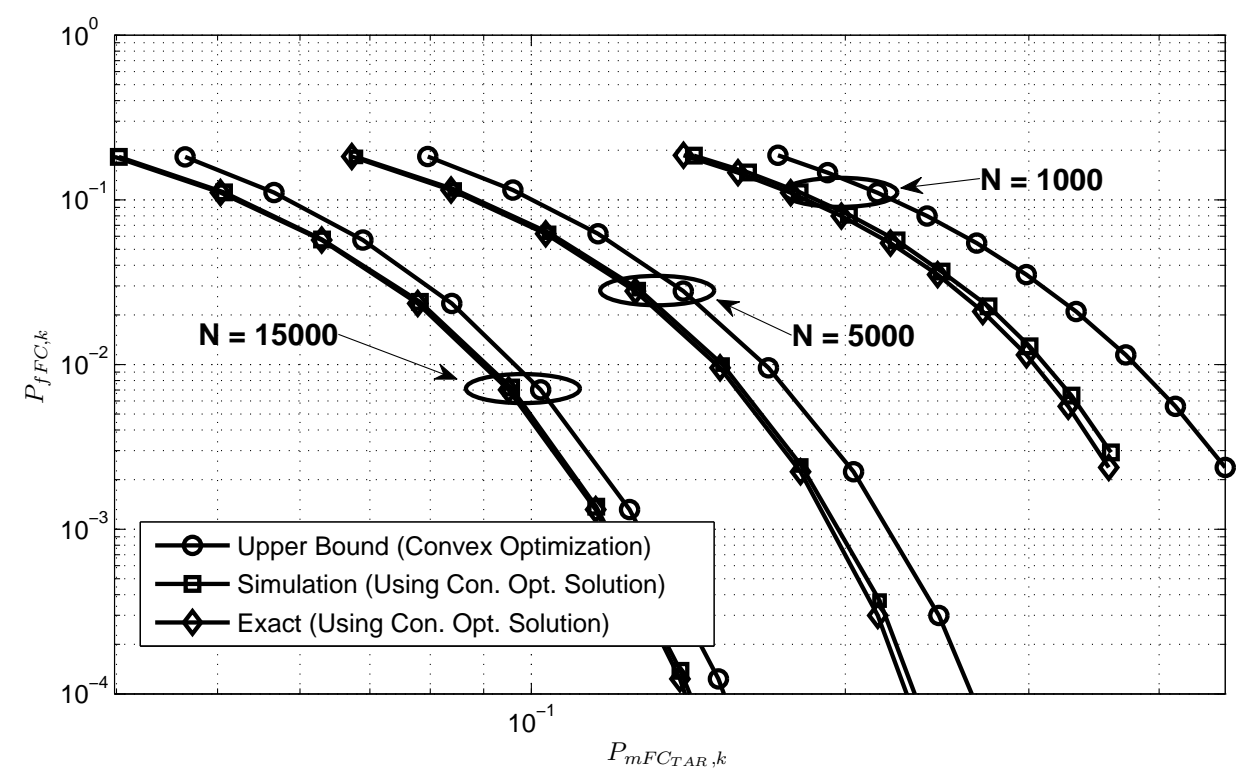

Figure 5.1: ROC for $\gamma_{R, k}=-6 \mathrm{~dB}$ with 1 sensor and three different values of $N$. The convex optimal curves were obtained using the solutions of (3.29) via (3.16) and (3.15). The exact and simulation curves were obtained with the solutions of (3.29) in exact expressions (3.10) and (3.11), and for the simulation of $10^{6}$ realizations of noises and sensing channels, respectively.

false alarm probability, convex optimization methods would no longer apply and we would have to rely on less efficient and more time-consuming methods, such as exhaustive search.

In Fig. 5.2, we see a decrease in performance when we optimize once based on the variance of the reporting channel, as expected. The looseness of the upper bound when optimizing once is comparable to the upper bound looseness when optimizing for the instantaneous reporting channel and averaging over many channel realizations. If we can accept the computational and communications overhead of optimizing more often, then we can achieve a measureable gain in performance. However, if the overhead becomes cumbersome, then optimizing less often becomes a viable alternative.

In both Figs. 5.1 and 5.2, we see a small difference between the curve(s) obtained from the exact expressions and the simulation curve(s), due to the Gaussian 


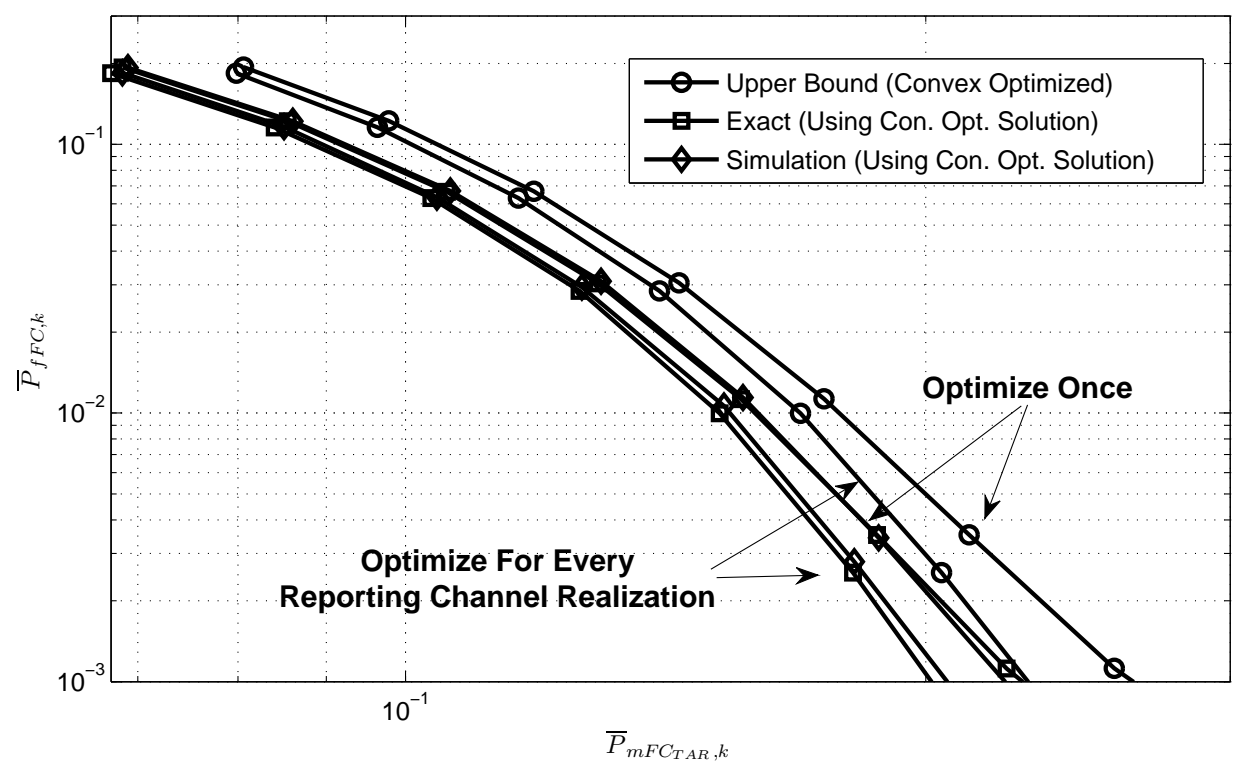

Figure 5.2: $\mathrm{ROC}$ for $\bar{\gamma}_{R, k}=-6 \mathrm{~dB}$ with 1 sensor and $N=5000$. One set of curves was obtained by optimizing once, based on $\bar{\gamma}_{R, k}$, by solving (3.30) via (3.28) and (3.27), then using the solutions of (3.30) in exact expressions (3.25) and (3.26), and finally the simulation of $10^{6}$ realizations of noise and sensing channels. The second set was obatined by optimizing for $10^{4}$ reporting channel realizations by solving (3.29) via (3.16) and (3.15), then using the solutions of (3.29) in exact expressions (3.10) and (3.11), and finally the simulation of 100 realizations of noise and sensing channels for each reporting channel realization.

approximation of the local energy detector decision variables and the rounding of the optimal $N_{R, k}$ to the nearest integer for simulation. Fig. 5.1 shows that this difference shrinks with increasing $N$, as expected from the central limit theorem. We also see that low $P_{f F C, k}$ is more readily achievable than low $P_{m F C, k}$, as the lower bounds on $\tau_{k}$ (i.e., (3.17) and (3.19)) are tighter constraints than the upper bound (i.e., (3.21)). This implies that the design of a multi-sensor network will favor a rule that multiplies missed detection probabilities and sums false alarm probabilities (i.e., OR-rule). 


\subsection{Multi-Sensor Results and Discussion}

Unless otherwise noted, results in this section are for a simulated secondary network with $K=4$ sensors. For this value of $K$, it is easy to show that constraints (4.7) and (4.8) can be ignored for all rules. For all sensors, $\sigma_{n, k}^{2}=1$ and $\sigma_{z, k}^{2}=1$, and we define $\bar{\gamma}_{\mathbf{S}}=[-7,-8,-9,-7] \mathrm{dB}$ and $\gamma_{\mathbf{R}}=[-6,0,-4,-2] \mathrm{dB}$, so that the sensors have unique pairs of sensing and reporting SNRs. Due to similarity of the results, we do not include optimizing for the average Rayleigh-faded reporting channels, $\bar{\gamma}_{\mathbf{R}}$. Such results would only serve to further illustrate the loses due to optimizing with less information.

All figures in this section show upper bounds obtained using the solutions of the convex problem (4.16), where the upper bound $P_{f F C}$ 's are calculated from (4.5). Curves are also shown that were obtained using the solutions of (4.16) in the exact expressions (3.10) and (3.11), and then combining the single-sensor probabilities with (4.1).

\subsubsection{Comparison of Fusion Rules}

Fig. 5.3 shows the ROCs for the secondary network using all possible $M$-out-of- $K$ rules when $K=4$. In addition to the aforementioned curves, it shows curves obtained by using the solutions of (4.16) in the simulation of $10^{6}$ realizations of noises and sensing channels. Furthermore, for OR-rule $(M=1)$ it shows a curve obtained by solving (4.11) with the branch-and-reduce method as a direct comparison to the suboptimal convex approach.

In Fig. 5.3, we see that OR-rule is generally superior to all other rules and that solving (4.16) yields performance identical to solving (4.11) for OR-rule, i.e. our suboptimal convex problem yields the same solutions as applying the branchand-reduce method. The overall performance for AND-rule is weaker due to high missed detection probabilities; we can only achieve $P_{m F C_{T A R}} \geq 0.3$ since the missed detection performance of AND-rule $(M=K=4)$ is derived from the addition of the single-sensor $P_{m F C, k}$ 's, which in turn are constrained by the lower bounds on $\tau_{k}$ (i.e., via (3.17) and (3.19)). The performance of rules $M=2$ and $M=3$ lie in between that of OR-rule and AND-rule, as expected. The upper bounds on all rules, which formed the basis of the optimization, are reasonably close to the 


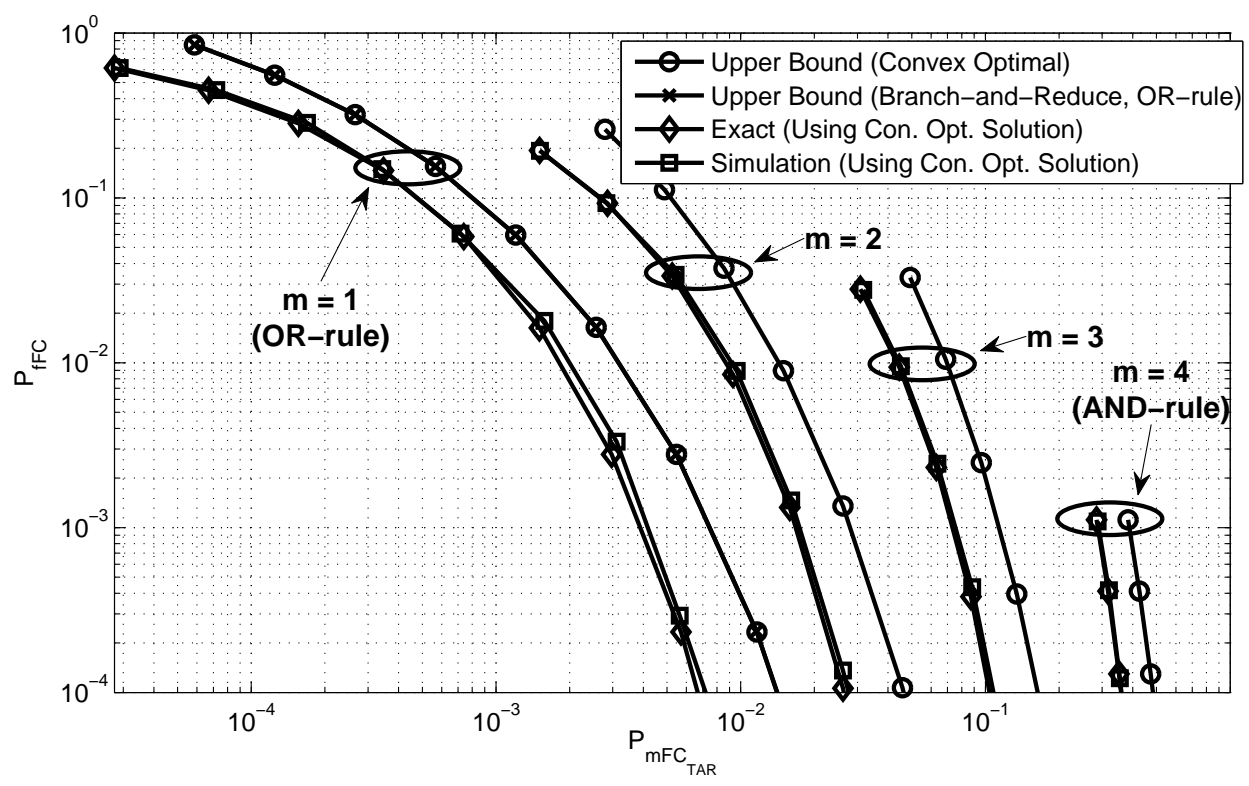

Figure 5.3: Secondary network ROC with 4 sensors and $N=5000$, using $M$ out-of- $K$ rules. The convex optimal and branch-and-reduce curves were obtained using the solutions of (4.16) and (4.11), respectively, where the latter was only solved for OR-rule. The exact and simulation curves were obtained with the solutions of (4.16) in exact expressions (3.10), (3.11), and (4.1), and for the simulation of $10^{6}$ realizations of noises and sensing channels, respectively.

exact performance. The looseness of the upper bounds has propagated from the looseness of the upper bounds of the single-sensor false alarm and missed detection probabilities.

As observed in the single-sensor case, there is a small difference between the curves obtained from the exact expressions and the simulation curves, again due to the Gaussian approximation of the energy detector decision variables and the rounding of the optimal $N_{R, k}$ 's to the nearest integers; the accuracy of the exact analytical curves can be arbitrarily improved by increasing $N$. Importantly, we observe that very good performance can be achieved using energy detection, especially using OR-rule, even though the sensing channels have $\bar{\gamma}_{S, k} \leq-7 \mathrm{~dB}, \forall k$. 


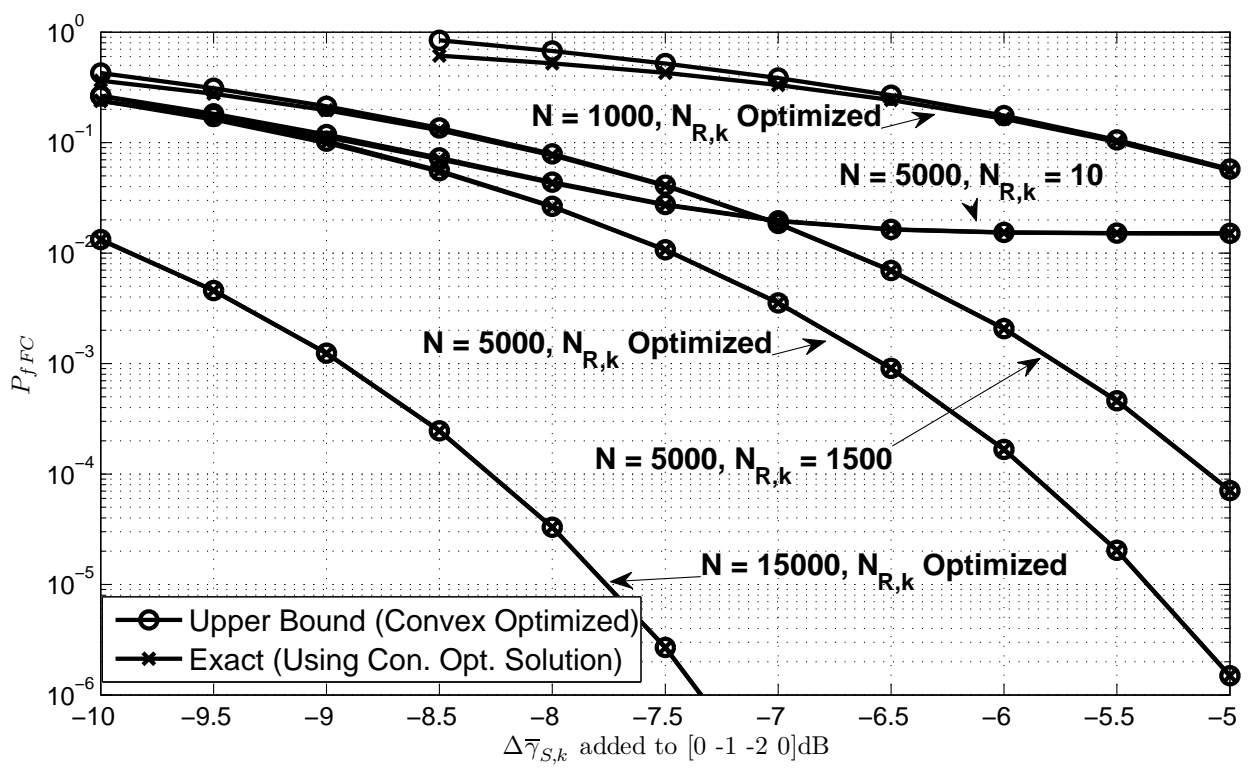

Figure 5.4: Effect of sensing channels on $P_{f F C}$ with 4 sensors using OR-rule and $P_{m F C_{T A R}}=0.005$. Reporting channel SNRs are $\gamma_{\mathbf{R}}=[-6,0,-4,-2] \mathrm{dB}$. For $N_{R, k}=10$ and $N_{R, k}=1500$, only the thresholds $\tau_{k}, \forall k$, are optimized.

\subsubsection{Sensitivity to Channel Quality with OR-Rule}

We next study the sensitivity of $P_{f F C}$ to the quality of the sensing and reporting channels when using OR-rule. At the same time, we consider the benefits of optimizing $N_{R, k}$ versus holding $N_{R, k}$ constant, since the current literature has not optimized $N_{R, k}$. When we keep $N_{R, k}$ constant, we only optimize the thresholds $\tau_{k}, \forall k$. We note that attempting to optimize $N_{R, k}$ while keeping $\tau_{k}$ constant significantly restricts performance so we do not consider it in this thesis.

Figs. 5.4 and 5.5 show the sensitivity of $P_{f F C}$ using OR-rule to the quality of the sensing and reporting channels, respectively, for $P_{m F C_{T A R}}=0.005$. We note that, because of the upper-bounding, the exact missed detection probabilities are $P_{m F C}<0.005$. Both figures show that a wide operating range exists where the false alarm probability can be guaranteed to be below $1 \%$ when $N=5000$. Comparative curves show results for $N_{R, k}=10$ and $N_{R, k}=1500, \forall k$. For Fig. 5.4, we also have curves optimizing $N_{R, k}$ for different interval length $N$ where we set $N_{R_{\max }}=0.3 \mathrm{~N}$ 


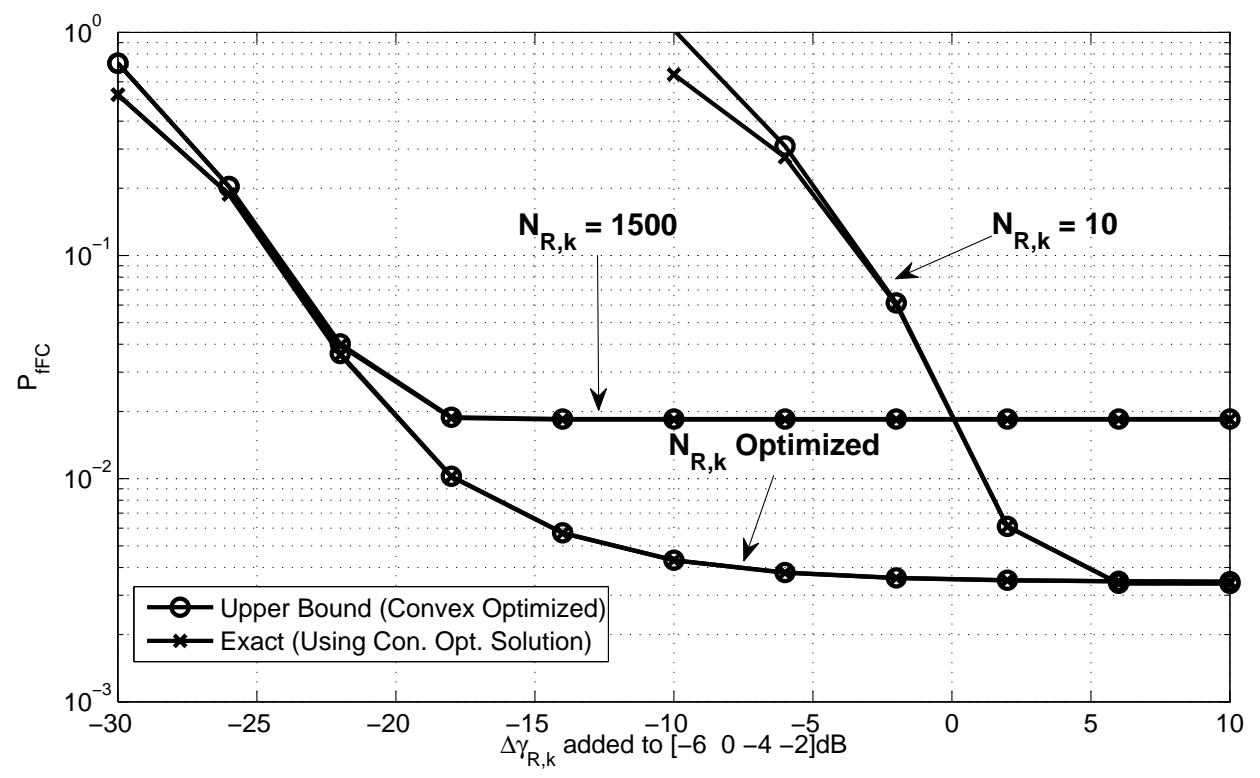

Figure 5.5: Effect of reporting channels on $P_{f F C}$ with 4 sensors and $N=$ 5000, using OR-rule, and $P_{m F C_{T A R}}=0.005$. Sensing channel SNRs are $\bar{\gamma}_{\mathbf{S}}=[-7,-8,-9,-7] \mathrm{dB}$. For $N_{R, k}=10$ and $N_{R, k}=1500$, only the thresholds $\tau_{k}, \forall k$, are optimized.

and $N_{S_{\max }}=N-1$.

In Fig. 5.4, we decrease the sensing SNRs from base values $\bar{\gamma}_{\mathbf{S}}=[0,-1,-2,0]$ dB. Allowing $N_{R, k}$ to be optimized when $N=5000$ permits significantly lower false alarm probabilities than when using $N_{R, k}=10$ or $N_{R, k}=1500$ for the majority of the considered range of sensing SNRs. When the sensing channels are particularly weak, i.e., $\bar{\gamma}_{\mathbf{S}}<[-9,-10,-11,-9] \mathrm{dB}$, we see that using $N_{R, k}=10$ is comparable to using the optimal $N_{R, k}$. Thus, as sensing channels weaken, it is beneficial to sacrifice reporting time slots to allow more time slots for sensing. Using $N_{R, k}=$ 1500 is not advised for the range of sensing channel SNRs considered in Fig. 5.4, since the reporting channels are relatively strong. When the sensing channels are relatively strong, having $N_{R, k}=1500$ does outperform $N_{R, k}=10$, so we see that the bottleneck in performance shifts from the sensing to the reporting channels. Still, significantly improved $P_{m F C}$ and $P_{f F C}$ are possible by optimizing $N_{R, k}$. For example, we can achieve $P_{f F C} \approx 1.5 \times 10^{-6}$ when $\bar{\gamma}_{\mathrm{S}}=[-5,-6,-7,-5] \mathrm{dB}$, which 
is almost two orders of magnitude lower than if we set $N_{R, k}=1500$ for the same sensing channels. Finally, the size of $N$ is also a major factor when optimizing $N_{R, k}$; significantly lower false alarm probabilities are achievable when $N=15000$, whereas performance when $N=1000$ is poor for the considered range of sensing SNRs due to the aggressive $P_{m F C_{T A R}}$.

In Fig. 5.5, we vary the reporting channels from base values $\gamma_{\mathbf{R}}=[-6,0,-4$, -2]dB. Optimizing $N_{R, k}$ allows lower $P_{f F C}$ than when setting $N_{R, k}=10$ or $N_{R, k}=$ 1500. When $N_{R, k}=10$, we see a sharp performance deterioration as the reporting channels weaken; the effective reporting SNR is too low for the reports of the sensors to reach the FC. Furthermore, when $\gamma_{\mathbf{R}}<[-29,-23,-27,-26] \mathrm{dB}$, $N_{R, k}=1500$ becomes the optimal value for all sensors. However, as reporting channels improve, the excessive reporting with $N_{R, k}=1500$ creates a bottleneck in performance that limits $P_{f F C}$ to almost an order of magnitude lower than when using the optimal $N_{R, k}$.

Figs. 5.4 and 5.5 both show that optimizing $\tau_{k}$ and $N_{R, k}$ enable a reasonable operating range with an aggressive missed detection target probability. Poor sensing or reporting channels can be mitigated to maintain network performance. The benefits of optimizing $N_{R, k}$ versus holding $N_{R, k}$ constant are substantial.

\subsubsection{Sensitivity to Channel Quality with Other Rules}

We now briefly study the sensitivity of $P_{f F C}$ to the quality of the sensing and reporting channels when not using OR-rule. Specifically, we consider the case of $M=2$.

Figs. 5.6 and 5.7 show the sensitivity of $P_{f F C}$ using $M=2$ to the quality of the sensing and reporting channels, respectively, for $P_{m F C_{T A R}}=0.005$. The setup is identical to that used for Figs. 5.4 and 5.5 with OR-rule, respectively, except that in Fig. 5.7 the sensing channel SNRs are $\bar{\gamma}_{\mathrm{S}}=[-5,-6,-7,-5] \mathrm{dB}$ in order to enable false alarm probabilities that are comparable to those in Fig. 5.5.

In Fig. 5.6, we decrease the sensing SNRs from base values $\bar{\gamma}_{\mathbf{S}}=[0,-1,-2,0]$ dB. As when using OR-rule, allowing $N_{R, k}$ to be optimized when $N=5000$ permits significantly lower false alarm probabilities than when using $N_{R, k}=10$ or $N_{R, k}=1500$ for the majority of the considered range of sensing SNRs. Overall, we 


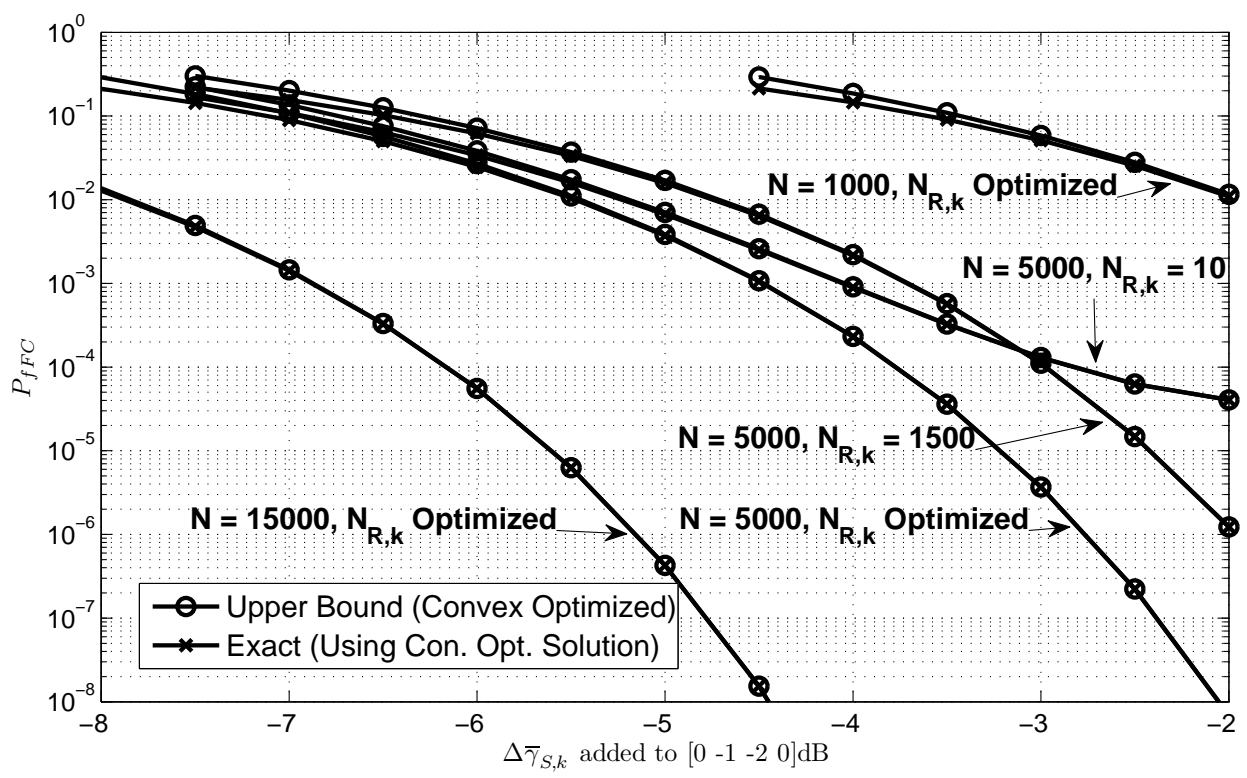

Figure 5.6: Effect of sensing channels on $P_{f F C}$ with 4 sensors using $M=2$ and $P_{m F C_{T A R}}=0.005$. Reporting channel SNRs are $\gamma_{\mathbf{R}}=[-6,0,-4,-2] \mathrm{dB}$. For $N_{R, k}=10$ and $N_{R, k}=1500$, only the thresholds $\tau_{k}, \forall k$, are optimized.

observe the same general trends for using $M=2$ as we did with $M=1$; we can mitigate the effects of weaker sensing channels by dedicating more time to sensing, we can take advantage of stronger sensing channels by dedicating more time to reporting, and increasing the total number of time slots $N$ enables an overall performance improvement. By comparing Fig. 5.6 with Fig. 5.4, we observe that a weak sensing channel obstructs performance more easily when $M=2$ than when $M=1$. This is as expected from the results in Fig. 5.3, where OR-rule is shown to be generally superior to the other rules.

In Fig. 5.7, we vary the reporting channels from base values $\gamma_{\mathbf{R}}=[-6,0,-4$, -2 ]dB. Once again, the general result trends are the same as those shown in Fig. 5.5, optimizing $N_{R, k}$ allows lower $P_{f F C}$ than when setting $N_{R, k}=10$ or $N_{R, k}=$ 1500. When $N_{R, k}=10$, we see a sharp performance deterioration as the reporting channels weaken, so the number of reporting time slots has become a major performance bottleneck. As noted previously, we used stronger sensing channel SNRs in order to observe false alarm probabilities comparable to those in Fig. 5.5; OR-rule 


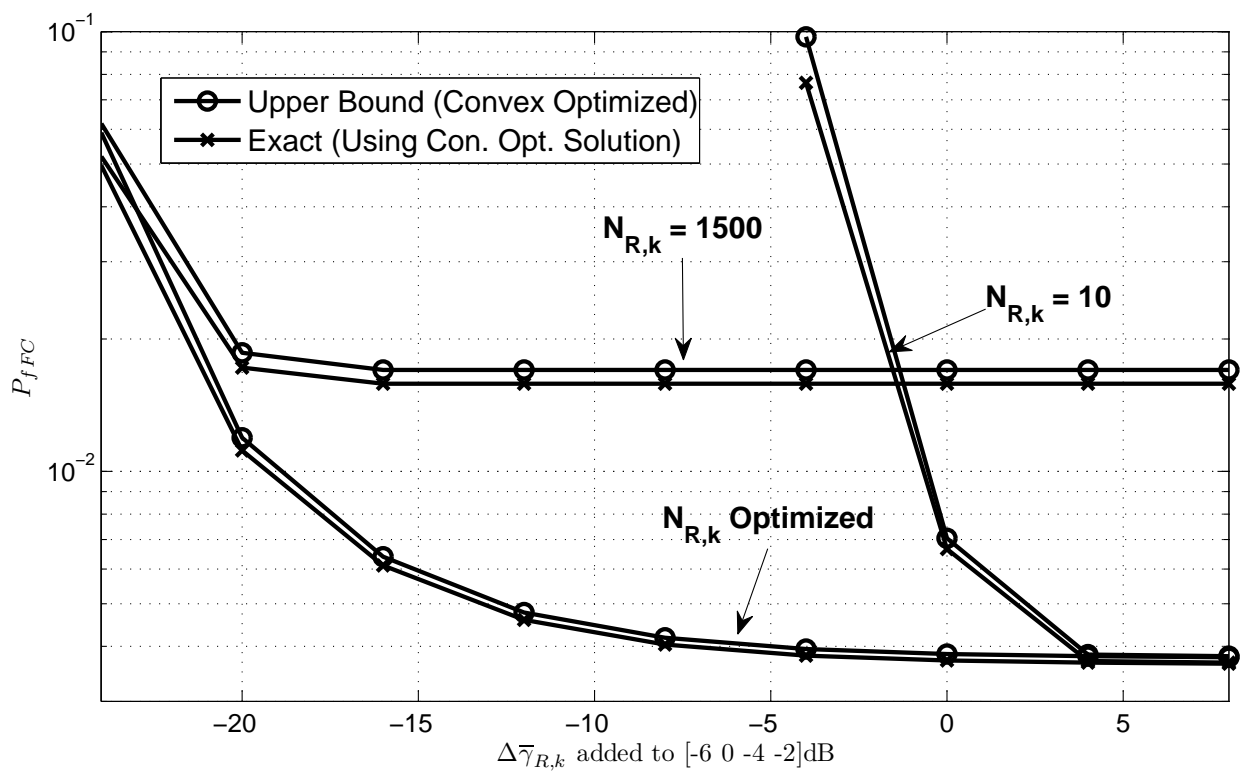

Figure 5.7: Effect of reporting channels on $P_{f F C}$ with 4 sensors and $N=$ 5000, using $M=2$, and $P_{m F C_{T A R}}=0.005$. Sensing channel SNRs are $\bar{\gamma}_{\mathbf{S}}=$ $[-5,-6,-7,-5] \mathrm{dB}$. For $N_{R, k}=10$ and $N_{R, k}=1500$, only the thresholds $\tau_{k}, \forall k$, are optimized.

has once again shown to be superior in this framework. 


\section{Chapter 6}

\section{Conclusions and Future Work}

\subsection{Conclusions}

In this thesis, we considered the problem of cooperative spectrum sensing for cognitive radio. The secondary network optimized the decision thresholds at the SUs and the division between time slots used for sensing the PU and time slots used for reporting the sensing results to the FC. We selected the network probabilities of false alarm and missed detection as our performance metrics. We derived these probabilities for a single-sensor network and then found bounds to facilitate convex optimization techniques. The analysis was extended to multi-sensor networks using $M$-out-of- $K$ rules. Using OR-rule, we represented the multi-sensor network optimization problem as a generalized convex multiplicative problem. We then relaxed the problem to formulate a convex suboptimal problem that usually yields the same results. The relaxation was then generalized to maintain convexity for any $M$-out-of- $K$ rule. Furthermore, we showed that we could optimize for the average reporting channel gains instead of the instantaneous ones if we were willing to accept a decrease in sensing performance. Simulation results showed that our convex upper bounds were close to the exact analytical performance, and that joint optimization of thresholds and sensing/reporting time slots enables very good sensing performance. 


\subsection{Future Work}

The following areas are identified as potential interesting extensions for the work in this thesis:

- Investigate scenarios with larger numbers of SUs where some may have sensing or reporting channel gains that are so weak that they should be explicitly omitted from optimization.

- Account for primary networks that have more than one transmitting PU. Even if only one PU transmits at a time, the SUs would have different channels to each PU, likely with different channel gains.

- Extend our approach to the case of imperfect channel estimation by accounting for additional performance loss due to the imperfect estimation of both the sensing channel variances and the instantaneous reporting channels.

- Seek improved codes for the reporting channel. Our implementation applied a basic repetition code. The sensing reports could be quantized at the cost of reporting SNR. It is unknown whether convexity would be maintained, especially since the FC would then need to implement soft decision combining. 


\section{Bibliography}

[1] J. Mitola and G. Q. Maguire. Cognitive radio: Making software radios more personal. IEEE Pers. Comm., 6(4):13-18, Aug. 1999. $\rightarrow$ pages 1

[2] S. Haykin. Cognitive radio: Brain-empowered wireless communications. IEEE J. Sel. Areas Commun., 23(2):201-220, Feb. 2005. $\rightarrow$ pages 1

[3] FCC. Spectrum Policy Task Force. Rep. ET Docket no. 02-135, Nov. 2002. $\rightarrow$ pages 2

[4] IEEE Std 802.22-2011, IEEE Standard for information technology Telecommunications and information exchange between systems wireless regional area networks (WRAN) - specific requirements part 22: Cognitive wireless RAN medium access control (MAC) and physical layer (PHY) specifications: Policies and procedures for operation in the tv bands, Jul. 2011. $\rightarrow$ pages 2

[5] D. Cabric, S. M. Mishra, and R. W. Brodersen. Implementation issues in spectrum sensing for cognitive radios. In Proc. ACSSC 2004, pages 772-776, Nov. 2004. $\rightarrow$ pages 2,3

[6] Ian F. Akyildiz, Brandon F. Lo, and Ravikumar Balakrishnan. Cooperative spectrum sensing in cognitive radio networks: A survey. Physical Communication, 4(1):40-62, Mar. 2011. $\rightarrow$ pages 2, 3, 4

[7] S. Atapattu, C. Tellambura, and H. Jiang. Energy detection based cooperative spectrum sensing in cognitive radio networks. IEEE Trans. Wireless Comm., 10(4):1232-1241, Apr. 2011. $\rightarrow$ pages 3, 4, 13

[8] Ying-Chang Liang, Yonghong Zeng, Edward C. Y. Peh, and Anh Tuan Hoang. Sensing-throughput tradeoff for cognitive radio networks. IEEE Trans. Wireless Comm., 7(4):1326-1337, Apr. 2008. $\rightarrow$ pages 5 
[9] Wei Zhang, Ranjan K. Mallik, and Khaled Ben Letaief. Optimization of cooperative spectrum sensing with energy detection in cognitive radio networks. IEEE Trans. Wireless Comm., 8(12):5761-5766, Dec. 2009. $\rightarrow$ pages 5

[10] Jun Ma, Guodong Zhao, and Ye Li. Soft combination and detection for cooperative spectrum sensing in cognitive radio networks. IEEE Trans. Wireless Comm., 7(11):4502-4507, Nov. 2008. $\rightarrow$ pages 3, 6

[11] Zhi Quan, Shuguang Cui, Ali H. Sayed, and H. V. Poor. Optimal multiband joint detection for spectrum sensing in cognitive radio networks. IEEE Trans. Sig. Process., 57(3):1128-1140, Mar. 2009. $\rightarrow$ pages 5

[12] E. Peh and Ying-Chang Liang. Optimization for cooperative sensing in cognitive radio networks. In Proc. IEEE WCNC 2007, pages 27-32, Mar. 2007. $\rightarrow$ pages 5,25

[13] Woongsup Lee and Dong-Ho Cho. Enhanced spectrum sensing scheme in cognitive radio systems with MIMO antennae. IEEE Trans. Veh. Tech., 60(3):1072-1085, Mar. 2011. $\rightarrow$ pages 5, 19

[14] Seung-Jun Kim and Georgios B. Giannakis. Sequential and cooperative sensing for multi-channel cognitive radios. IEEE Trans. Sig. Proc., 58(8):4239-4253, Aug. 2010. $\rightarrow$ pages 5

[15] Tao Cui, Feifei Gao, and Arumugam Nallanathan. Optimization of cooperative spectrum sensing in cognitive radio. IEEE Trans. Veh. Tech., 60(4):1578-1589, May 2011. $\rightarrow$ pages

[16] Zhi Quan, Shuguang Cui, and Ali H. Sayed. Optimal linear cooperation for spectrum sensing in cognitive radio networks. IEEE J. Sel. Top. Sig.

Process., 2(1):28-40, Feb. 2008. $\rightarrow$ pages 3. 5

[17] P. K. Varshney. Distributed Detection and Data Fusion. Springer-Verlag, New York, 1997. $\rightarrow$ pages 4

[18] F. Moghimi, A. Nasri, and R. Schober. Lp-norm spectrum sensing for cognitive radio networks impaired by non-Gaussian noise. In Proc. IEEE GLOBECOM 2009, pages 1-6, Dec. 2009. $\rightarrow$ pages 4, 13, 19, 56

[19] N. C. Beaulieu and Y. Chen. Improved energy detectors for cognitive radios with randomly arriving or departing primary users. IEEE Sig. Proc. Letters, 17(10):867-870, Oct. 2010. $\rightarrow$ pages 10 
[20] K. B. Oldham, J. C. Myland, and J. Spanier. An Atlas of Functions with Equator, the Atlas Function Calculator. Springer, 2nd edition, 2008. $\rightarrow$ pages $14,16,46$

[21] G. T. F. de Abreu. Supertight algebraic bounds on the Gaussian Q-function. In Proc. ACSSC 2009, pages 948-951, Nov. 2009. $\rightarrow$ pages 16, 18, 44

[22] S. Boyd and L. Vandenberghe. Convex Optimization. Cambridge University Press, Cambridge, 2004. $\rightarrow$ pages 18, 20, 26, 49, 59

[23] R. Horn and C. Johnson. Matrix Analysis. Cambridge University Press, Cambridge, 1985. $\rightarrow$ pages 18,49

[24] C. A. Floudas and P. M. Pardalos. Encyclopedia of Optimization. Kluwer Academic Publishers, 2001. $\rightarrow$ pages 21, 24

[25] A. R. Elias-Fuste, A. Broquetas-Ibars, J. P. Antequera, and J. C. M. Yuste. CFAR data fusion center with inhomogeneous receivers. IEEE Trans. Aero. Elec. Sys., 28(1):276-285, Jan. 1992. $\rightarrow$ pages 22

[26] R. L. Graham, D. E. Knuth, and O. Patashnik. Concrete Mathematics. Addison-Wesley, Reading, MA, 1994. $\rightarrow$ pages 22, 23

[27] H. P. Benson. Simplicial branch-and-reduce algorithm for convex programs with a multiplicative constraint. J. Optim. Theory Appl., 145(2):213-233, 2010. $\rightarrow$ pages 24,25

[28] D. Tse and P. Viswanath. Fundamentals of Wireless Communication. Cambridge University Press, Cambridge, 2005. $\rightarrow$ pages 56 


\section{Appendix A}

\section{Proof of Theorem 1}

To upper-bound (3.3), we first convert (3.4) to an equivalent form using the relation $\operatorname{erf}(x)=1-2 Q(\sqrt{2} x)$ to obtain

$$
S=-2+2 Q(\sqrt{2} a)+4 Q(\sqrt{2} b)
$$

where

$$
\begin{aligned}
& a=\sqrt{\frac{N_{S, k}}{2}}\left(\frac{\tau_{k}}{\sigma_{n, k}^{2}}-1\right) \frac{1}{\sin \theta}-\frac{\sin \theta \sigma_{n, k}^{2}}{\sqrt{2 N_{S, k}} P_{t} \sigma_{h, k}^{2}}, \\
& b=\frac{\sin \theta \sigma_{n, k}^{2}}{\sqrt{2 N_{S, k}} P_{t} \sigma_{h, k}^{2}} .
\end{aligned}
$$

We observe that an upper bound on (A.1) results in an upper bound on (3.3). Considering tractability of the integral in (3.3), we propose the use of the recentlydeveloped "supertight bound" on the $Q$-function [21], defined as

$$
Q(c) \leq \frac{1}{50} \exp \left(-c^{2}\right)+\frac{1}{2(c+1)} \exp \left(\frac{-c^{2}}{2}\right) .
$$


Eq. (A.4) is tight for all values of $c$, and allows us to write

$$
\begin{aligned}
S \leq & -2+\frac{1}{25} \exp \left(-2 a^{2}\right)+\frac{1}{\sqrt{a}+1} \exp \left(-a^{2}\right) \frac{2}{25} \exp \left(-2 b^{2}\right) \\
& +\frac{2}{\sqrt{b}+1} \exp \left(-b^{2}\right) .
\end{aligned}
$$

Before plugging $S$ back into $I$, we further upper-bound some of its terms, as follows:

$$
\begin{aligned}
-2 a^{2} & \leq \frac{\sigma_{n, k}^{2}}{P_{t} \sigma_{h, k}^{2}}\left(\frac{\tau_{k}}{\sigma_{n, k}^{2}}-1\right)-\frac{\sin ^{2} \theta}{N_{S, k}} \frac{\sigma_{n, k}^{4}}{P_{t}^{2} \sigma_{h, k}^{4}}-N_{S, k}\left(\frac{\tau_{k}}{\sigma_{n, k}^{2}}-1\right)^{2}, \\
\frac{1}{\sqrt{a}+1} & \leq \frac{\sqrt{N_{S, k}} P_{t} \sigma_{h, k}^{2}}{N_{S, k} P_{t} \sigma_{h, k}^{2}\left(\frac{\tau_{k}}{\sigma_{n, k}^{2}}-1\right)-\sigma_{n, k}^{2}+\sqrt{N_{S, k}} P_{t} \sigma_{h, k}^{2}}, \\
\frac{1}{\sqrt{b}+1} & \leq 1+\sin \theta\left(\frac{\sqrt{N_{S, k}} P_{t} \sigma_{h, k}^{2}}{\sigma_{n, k}^{2}+\sqrt{N_{S, k}} P_{t} \sigma_{h, k}^{2}}-1\right),
\end{aligned}
$$

where for (A.6) we used the fact that $1 \geq \sin ^{2} \theta$, for (A.8) we used a linear approximation with respect to $\sin \theta$ (which is an upper bound since $1 /(\sqrt{b}+1)$ is convex with respect to $\sin \theta$ ), and for (A.7) to be satisfied we impose the constraint

$$
\frac{\sigma_{n, k}^{2}}{N-N_{R, k}}-P_{t} \sigma_{h, k}^{2}\left(\frac{\tau_{k}}{\sigma_{n, k}^{2}}-1\right)<0 .
$$

This constraint is convex with respect to both $N_{R, k}$ and $\tau_{k}$. I is now bounded by

$$
I \leq \frac{1}{\sqrt{2 \pi N_{S, k}}} \exp \left(-\frac{\tau_{k}-\sigma_{n, k}^{2}}{P_{t} \sigma_{h, k}^{2}}\right) \int_{0}^{\frac{\pi}{2}} S_{1} d \theta
$$


where

$$
\begin{aligned}
S_{1}= & -2 \beta_{1}+2\left(\frac{\sqrt{N_{S, k}} P_{t} \sigma_{h, k}^{2}}{\sigma_{n}^{2}+\sqrt{N_{S, k}} P_{t} \sigma_{h, k}^{2}}-1\right) \beta_{2}^{2} \\
& +\frac{2 \beta_{3}}{25}+\frac{\beta_{3}}{25} \exp \left(\frac{\sigma_{n, k}^{2}}{P_{t} \sigma_{h, k}^{2}}\left(\frac{\tau_{k}}{\sigma_{n, k}^{2}}-1\right)-N_{S, k}\left(\frac{\tau_{k}}{\sigma_{n, k}^{2}}-1\right)^{2}\right) \\
& +\left[2+\frac{\sqrt{N_{S, k}} P_{t} \sigma_{h, k}^{2}}{N_{S, k} P_{t} \sigma_{h, k}^{2}\left(\frac{\tau_{k}}{\sigma_{n, k}^{2}}-1\right)-\sigma_{n}^{2}+\sqrt{N_{S, k}} P_{t} \sigma_{h, k}^{2}}\right. \\
& \left.\times \exp \left(\frac{1}{2} \frac{\sigma_{n, k}^{2}}{P_{t} \sigma_{h, k}^{2}}\left(\frac{\tau_{k}}{\sigma_{n, k}^{2}}-1\right)-\frac{N_{S, k}}{2}\left(\frac{\tau_{k}}{\sigma_{n, k}^{2}}-1\right)^{2}\right)\right] \beta_{2}, \\
\beta_{1}= & \sin \theta \exp \left(\frac{\sin ^{2} \theta}{2 N_{S, k}} \frac{\sigma_{n, k}^{4}}{P_{t}^{2} \sigma_{h, k}^{4}}\right), \\
\beta_{2}= & \sin \theta, \\
\beta_{3}= & \sin \theta \exp \left(-\frac{\sin ^{2} \theta}{2 N_{S, k}} \frac{\sigma_{n, k}^{4}}{P_{t}^{2} \sigma_{h, k}^{4}}\right) .
\end{aligned}
$$

The integral over $S_{1}$ can be computed in closed form. The integration of $\beta_{2}^{2}$ and $\beta_{2}$ from 0 to $\frac{\pi}{2}$ yields $\frac{\pi}{4}$ and 1 , respectively. The integration of $\beta_{1}$ and $\beta_{3}$ can be solved by exploiting the identity $\sin ^{2} \theta=1-\cos ^{2} \theta$ and the substitution $t=p \cos \theta$, where $p$ is the magnitude of the exponential term that is independent of $\theta$. This leads to

$$
\begin{aligned}
\int_{0}^{\frac{\pi}{2}} \sin \theta \exp \left(p^{2} \sin ^{2} \theta\right) d \theta & =\exp \left(a^{2}\right) \frac{\sqrt{\pi}}{2 a} \operatorname{erf}(p) \\
\int_{0}^{\frac{\pi}{2}} \sin \theta \exp \left(-p^{2} \sin ^{2} \theta\right) d \theta & =\exp \left(-a^{2}\right) \frac{\sqrt{\pi}}{2 a} \operatorname{erfi}(p)
\end{aligned}
$$

where [20]

$$
\begin{aligned}
\operatorname{erf}(p) & =\frac{2}{\sqrt{\pi}} \int_{0}^{p} \exp \left(-t^{2}\right) d t \\
\operatorname{erfi}(p) & =\frac{2}{\sqrt{\pi}} \int_{0}^{p} \exp \left(t^{2}\right) d t
\end{aligned}
$$


Thus, $I$ is bounded in closed form by

$$
\begin{aligned}
I \leq & \frac{1}{\sqrt{2 \pi N_{S, k}}} \frac{\sigma_{n, k}^{2}}{P_{t} \sigma_{h, k}^{2}} \exp \left(-\frac{\tau_{k}-\sigma_{n, k}^{2}}{P_{t} \sigma_{h, k}^{2}}\right)[2 \\
& -\frac{\sqrt{2 \pi N_{S, k}} P_{t} \sigma_{h, k}^{2}}{\sigma_{n, k}^{2}} \exp \left(\frac{1}{2 N_{S, k}} \frac{\sigma_{n, k}^{4}}{P_{t}^{2} \sigma_{h, k}^{4}}\right) \operatorname{erf}\left(\frac{1}{\sqrt{2 N_{S, k}}} \frac{\sigma_{n, k}^{2}}{P_{t} \sigma_{h, k}^{2}}\right) \\
& +\frac{1}{25}\left(2+\exp \left(\frac{\sigma_{n, k}^{2}}{P_{t} \sigma_{h, k}^{2}}\left(\frac{\tau_{k}}{\sigma_{n, k}^{2}}-1\right)-N_{S, k}\left(\frac{\tau_{k}}{\sigma_{n, k}^{2}}-1\right)^{2}\right)\right) \\
& \times \frac{\sqrt{\pi N_{S, k}} P_{t} \sigma_{h, k}^{2}}{\sqrt{2} \sigma_{n, k}^{2}} \exp \left(-\frac{1}{2 N_{S, k}} \frac{\sigma_{n, k}^{4}}{P_{t}^{2} \sigma_{h, k}^{4}}\right) \operatorname{erfi}\left(\frac{1}{\sqrt{2 N_{S, k}}} \frac{\sigma_{n, k}^{2}}{P_{t} \sigma_{h, k}^{2}}\right) \\
& \frac{\sqrt{N_{S, k}} P_{t} \sigma_{h, k}^{2}}{N_{S, k} P_{t} \sigma_{h, k}^{2}\left(\frac{\tau_{k}}{\sigma_{n, k}^{2}}-1\right)-\sigma_{n, k}^{2}+\sqrt{N_{S, k}} P_{t} \sigma_{h, k}^{2}} \\
& \times \exp \left(\frac{1}{2} \frac{\sigma_{n, k}^{2}}{P_{t} \sigma_{h, k}^{2}}\left(\frac{\tau_{k}}{\sigma_{n, k}^{2}}-1\right)-\frac{N_{S, k}}{2}\left(\frac{\tau_{k}}{\sigma_{n, k}^{2}}-1\right)^{2}\right) \\
& \left.+\frac{\pi}{2}\left(\frac{\sqrt{N_{S, k}} P_{t} \sigma_{h, k}^{2}}{\sigma_{n, k}^{2}+\sqrt{N_{S, k}} P_{t} \sigma_{h, k}^{2}}-1\right)\right] .
\end{aligned}
$$

Eq. (A.19) can be evaluated quickly. However, it is not necessarily jointly convex with respect to $\tau_{k}$ and $N_{R, k}$; additional bounding is required.

First, the placements of $N_{S, k}$ in (A.19) (especially inside of the exponentials and square roots) make analysis of convexity with respect to $N_{R, k}$ cumbersome, if not impossible. To address this issue, we bound most of the $N_{S, k}$ terms to either $N_{S_{\min }}$ or $N_{S_{\max }}$ (whichever is appropriate to upper bound).

Second, the summation term with the error function, once multiplied by the outside exponential, results in a term that is concave with respect to $\tau_{k}$. We linearize and upper-bound this term using the first degree Taylor series approximation at $\tau_{k}=\sigma_{n, k}^{2}$. 
Performing these approximations leads to

$$
\begin{aligned}
I \leq & \beta_{5}+\frac{1}{25} \operatorname{erfi}\left(\frac{1}{\sqrt{2 N_{S_{\min }}}} \frac{\sigma_{n, k}^{2}}{P_{t} \sigma_{h, k}^{2}}\right) \exp \left(-\frac{1}{2 N_{S_{\max }}} \frac{\sigma_{n, k}^{4}}{P_{t}^{2} \sigma_{h, k}^{4}}\right)\left[2 \exp \left(-\frac{\tau_{k}-\sigma_{n, k}^{2}}{P_{t} \sigma_{h, k}^{2}}\right)\right. \\
& \left.+\exp \left(-N_{S_{\min }}\left(\frac{\tau_{k}}{\sigma_{n, k}^{2}}-1\right)^{2}\right)\right]+ \\
& \frac{1}{\sqrt{2 \pi}} \frac{\sigma_{n, k}^{2}}{N_{S_{\min }} P_{t} \sigma_{h, k}^{2}\left(\frac{\tau_{k}}{\sigma_{n, k}^{2}}-1\right)-\sigma_{n, k}^{2}+\sqrt{N_{S_{\min }}} P_{t} \sigma_{h, k}^{2}} \\
& \times \exp \left(-\frac{1}{2} \frac{\tau_{k}-\sigma_{n, k}^{2}}{P_{t} \sigma_{h, k}^{2}}-\frac{N_{S_{\min }}}{2}\left(\frac{\tau_{k}}{\sigma_{n, k}^{2}}-1\right)^{2}\right) \\
& +\frac{1}{\sqrt{2 \pi N_{S, k}}} \frac{\sigma_{n, k}^{2}}{P_{t} \sigma_{h, k}^{2}} \exp \left(-\frac{\tau_{k}-\sigma_{n, k}^{2}}{P_{t} \sigma_{h, k}^{2}}\right)\left(2+\frac{\pi}{2}\left(\frac{\sqrt{N_{S_{\max }}} P_{t} \sigma_{h, k}^{2}}{\sigma_{n, k}^{2}+\sqrt{N_{S_{\max }}} P_{t} \sigma_{h, k}^{2}}-1\right)\right),
\end{aligned}
$$

where

$$
\beta_{5}=\left(\frac{\tau_{k}-\sigma_{n, k}^{2}}{P_{t} \sigma_{h, k}^{2}}-1\right) \exp \left(\frac{1}{2 N_{S_{\max }}} \frac{\sigma_{n, k}^{4}}{P_{t}^{2} \sigma_{h, k}^{4}}\right) \operatorname{erf}\left(\frac{1}{\sqrt{2 N_{S, k}}} \frac{\sigma_{n, k}^{2}}{P_{t} \sigma_{h, k}^{2}}\right) .
$$

Observe that the only values of $N_{S, k}$ that are not bounded in (A.20) and (A.21) are the one inside the error function and the one in the denominator of the last summation term. $\beta_{5}$ in (A.21) is concave with respect to $N_{R, k}$ for $N_{R, k}>0$. We linearize and upper-bound this term using the first degree Taylor series approximation at $N_{R, k}=0$. The final result is (3.12), which upper-bounds $I$. 


\section{Appendix B}

\section{Proof of Convexity of Bound on (3.16)}

The convexity of $P_{f F C, k}$ as given in (3.16) can be proven by showing that its Hessian is positive semi-definite (PSD) [22]. The Hessian of (3.16) is not always PSD, but we will show that it is PSD over a convex region once we impose a set of additional constraints.

From [23], a matrix is PSD if and only if all of its principal minors are nonnegative. The minor $R_{\alpha \beta}$ of matrix $R$ is the determinant of the matrix formed by removing the rows of $R$ defined by the set $\alpha$ and the columns of $R$ defined by the set $\beta . R_{\alpha \beta}$ is a principal minor if $R$ is a square matrix and $\alpha=\beta$. In other words, we prove joint convexity of $P_{f F C, k}$ with respect to $\tau_{k}$ and $N_{R, k}$ by proving that

$$
\begin{aligned}
\frac{\partial^{2} P_{f F C, k}}{\partial \tau_{k}^{2}} & \geq 0, \\
\frac{\partial^{2} P_{f F C, k}}{\partial N_{R, k}^{2}} & \geq 0, \\
\frac{\partial^{2} P_{f F C}}{\partial \tau_{k}^{2}} \frac{\partial^{2} P_{f F C, k}}{\partial N_{R, k}^{2}}-\left(\frac{\partial^{2} P_{f F C, k}}{\partial \tau_{k} \partial N_{R, k}}\right)^{2} & \geq 0 .
\end{aligned}
$$


The individual second derivatives are

$$
\begin{aligned}
\frac{\partial^{2} P_{f F C, k}}{\partial \tau_{k}^{2}}= & \frac{\left(N-N_{R, k}\right)^{3 / 2}}{\sigma_{z, k}^{4} \sqrt{2 \pi}} x_{k} \exp \left(-x_{k}^{2} \frac{N-N_{R, k}}{2}\right) \\
\frac{\partial^{2} P_{f F C, k}}{\partial N_{R, k}^{2}}= & \frac{1}{4 \sqrt{2 \pi\left(N-N_{R, k}\right)}} x_{k} \exp \left(-x_{k}^{2} \frac{N-N_{R, k}}{2}\right)\left[\frac{1}{N-N_{R, k}}+x_{k}^{2}\right] \\
& +\frac{1}{2 \sqrt{N_{R, k} \pi \sigma_{z, k}^{2}}}\left|g_{k}\right| \exp \left(-\left|g_{k}\right|^{2} \frac{N_{R, k}}{\sigma_{z, k}^{2}}\right)\left[\frac{1}{2 N_{R, k}}+\frac{\left|g_{k}\right|^{2}}{\sigma_{z, k}^{2}}\right] \\
\frac{\partial^{2} P_{f F C, k}}{\partial \tau_{k} \partial N_{R, k}}= & \frac{1}{\sigma_{n, k}^{2} \sqrt{2 \pi}} \exp \left(-x_{k}^{2} \frac{N-N_{R, k}}{2}\right)\left[\frac{1}{2 \sqrt{N-N_{R, k}}}-\frac{\sqrt{N-N_{R, k}}}{2} x_{k}^{2}\right]
\end{aligned}
$$

where we used $d Q(w) / d w=-\exp \left(-w^{2} / 2\right) / \sqrt{2 \pi}$ and $x_{k}$ as defined in (3.13). To satisfy (B.1) and (B.2), we immediately obtain the following convex constraints by inspection:

$$
\begin{aligned}
& \sigma_{n, k}^{2}-\tau_{k} \leq 0 \\
& 1 \leq N_{R, k} \leq N
\end{aligned}
$$

Since we have previously defined $N_{S_{\min }}$, we have $N_{R_{\max }}=N-N_{S_{\min }}$. Therefore, (B.8) must be more tightly bound as

$$
1 \leq N_{R, k} \leq N_{R_{\max }}
$$

We observe that (B.5) has a positive term with an exponential that is different from those in (B.4) and (B.6). Therefore, we will ignore the unique term in (B.5) 
to satisfy (B.3). Thus, it is straightforward to show that

$$
\begin{aligned}
\frac{\partial^{2} P_{f F C, k}}{\partial \tau_{k}^{2}} \frac{\partial^{2} P_{f F C, k}}{\partial N_{R, k}^{2}}-\left(\frac{\partial^{2} P_{f F C, k}}{\partial \tau_{k} \partial N_{R, k}}\right)^{2} & \geq \frac{1}{2 \pi \sigma_{n}^{4}} \exp \left(-\left(\frac{\tau_{k}}{\sigma_{n, k}^{2}}-1\right)^{2}\left(N-N_{R, k}\right)\right) \\
& \times \underbrace{\left[\frac{3}{4}\left(\frac{\tau_{k}}{\sigma_{n, k}^{2}}-1\right)^{2}-\frac{1}{4\left(N-N_{R, k}\right)}\right]}_{\beta_{6}} .
\end{aligned}
$$

Non-negativity can then be guaranteed by making $\beta_{6}$ non-negative, or in other words imposing the convex constraint

$$
\left(\frac{\tau_{k}}{\sigma_{n, k}^{2}}-1\right)^{-2}+3 N_{R, k}-3 N \leq 0 .
$$

Thus, by imposing contraints to satisfy (B.1) to (B.3), we have defined a convex region where (3.16) is convex. 


\section{Appendix C}

\section{Proof of Convexity of Bound on (3.15)}

Analogously to the $P_{f F C, k}$ case in Appendix $\mathrm{B}$, for the convexity of $P_{m F C, k}$ as given in (3.15) we must prove that

$$
\begin{aligned}
\frac{\partial^{2} P_{m F C, k}}{\partial \tau_{k}^{2}} & \geq 0, \\
\frac{\partial^{2} P_{m F C, k}}{\partial N_{R, k}^{2}} & \geq 0, \\
\frac{\partial^{2} P_{m F C, k}}{\partial \tau_{k}^{2}} \frac{\partial^{2} P_{m F C, k}}{\partial N_{R, k}^{2}}-\left(\frac{\partial^{2} P_{m F C, k}}{\partial \tau_{k} \partial N_{R, k}}\right)^{2} & \geq 0 .
\end{aligned}
$$

The individual second derivatives of (3.15) are

$$
\begin{aligned}
& \frac{\partial^{2} P_{m F C, k}}{\partial \tau_{k}^{2}}=\frac{\partial^{2} I_{\text {bound }}}{\partial \tau_{k}^{2}} \\
& \frac{\partial^{2} P_{m F C, k}}{\partial N_{R, k}^{2}}=\frac{\left|g_{k}\right|}{4 \sqrt{\pi \sigma_{n}^{2} N_{R, k}}} \exp \left(-\left|g_{k}\right|^{2} \frac{N_{R, k}}{\sigma_{z, k}^{2}}\right)\left[\frac{1}{N_{R, k}}+\frac{2\left|g_{k}\right|^{2}}{\sigma_{z, k}^{2}}\right]+\frac{\partial^{2} I_{\text {bound }}}{\partial N_{R, k}^{2}}, \\
& \frac{\partial^{2} P_{m F C, k}}{\partial \tau_{k} \partial N_{R, k}}=\frac{\partial^{2} I_{\text {bound }}}{\partial \tau_{k} \partial N_{R, k}}
\end{aligned}
$$


where $I_{\text {bound }}$ is from (3.12). It can be shown that

$$
\begin{aligned}
& \frac{\partial^{2} I_{\text {bound }}}{\partial \tau_{k}^{2}}=\frac{C_{k}}{\sigma_{n}^{4}}\left[A_{k}^{2} \exp \left(-A_{k} x_{k}\right)+N_{S_{\text {min }}} \exp \left(-N_{S_{\min }} x_{k}^{2}\right)\left(2 N_{S_{\min }} x_{k}^{2}-1\right)\right] \\
& +\frac{A_{k}^{2} D_{k}}{\sigma_{n}^{4} \sqrt{N-N_{R, k}}} \exp \left(-A_{k} x_{k}\right)+\frac{A}{\sqrt{2 \pi} \sigma_{n}^{4}} \exp \left(-\frac{1}{2} A_{k} x_{k}-\frac{N_{S_{\min }}}{2} x_{k}^{2}\right) \\
& \times\left[\frac{2 N_{S_{\text {min }}}^{2}}{\left(N_{S_{\min }} x_{k}-A_{k}+\sqrt{N_{S_{\min }}}\right)^{3}}+\frac{2 A_{k} N_{S_{\min }}+N_{S_{\min }}^{2} x_{k}-N_{S_{\min }}^{3 / 2}}{\left(N_{S_{\min }} x_{k}-A_{k}+\sqrt{N_{S_{\min }}}\right)^{2}}\right. \\
& \left.+\frac{A_{k}^{2} / 4+A_{k} N_{S_{\text {min }}} x_{k}+N_{S_{\text {min }}}^{2} x_{k}^{2}}{\left(N_{S_{\min }} x_{k}-A_{k}+\sqrt{N_{S_{\min }}}\right)}\right] \text {, } \\
& \frac{\partial^{2} I_{\text {bound }}}{\partial N_{R, k}^{2}}=\frac{3 D_{k}}{4\left(N-N_{R, k}\right)^{5 / 2}} \exp \left(-A_{k} x_{k}\right), \\
& \frac{\partial^{2} I_{\text {bound }}}{\partial \tau_{k} \partial N_{R, k}}=\frac{A_{k}^{2}}{\sigma_{n, k}^{2} \sqrt{2 \pi} N_{S_{\max }^{3 / 2}}^{3}}-\frac{A_{k} D_{k}}{2 \sigma_{n, k}^{2}\left(N-N_{R, k}\right)^{3 / 2}} \exp \left(-A_{k} x_{k}\right) .
\end{aligned}
$$

Eq. (C.5) combined with (B.9) and (C.8) satisfies (C.2). Eq. (C.7) is still cumbersome to work with, but we can ignore a number of its components. To start, consider the terms

$$
\frac{2 A_{k} N_{S_{\text {min }}}+N_{S_{\min }}^{2} x_{k}-N_{S_{\min }}^{3 / 2}}{\left(N_{S_{\min }} x_{k}-A_{k}+\sqrt{N_{S_{\min }}}\right)^{2}}+\frac{A_{k}^{2} / 4+A_{k} N_{S_{\min }} x_{k}+N_{S_{\min }}^{2} x_{k}^{2}}{\left(N_{S_{\min }} x_{k}-A_{k}+\sqrt{N_{S_{\min }}}\right)}
$$

which include the only term of (C.7) that could be negative. By recalling constraint (A.9), we can show that (C.10) is non-negative if we also add the convex constraint

$$
x_{k}^{-2}-(1+\sqrt{2}) N_{S_{\min }} \leq 0
$$

which is more restrictive than (B.11), so we ignore (B.11). Thus, we ignore the term (C.10) in (C.7), and we have satisfied (C.1).

Finally, we show how (C.3) is satisfied. Again, by ignoring terms known to be 
non-negative, we write

$$
\begin{aligned}
& \frac{\partial^{2} P_{m F C, k}}{\partial \tau_{k}^{2}} \frac{\partial^{2} P_{m F C, k}}{\partial N_{R, k}^{2}}-\left(\frac{\partial^{2} P_{m F C, k}}{\partial \tau_{k} \partial N_{R, k}}\right)^{2} \geq \underbrace{\frac{A_{k}^{2} D_{k}}{\sigma_{n}^{4} \sqrt{N-N_{R, k}}} \exp \left(-A_{k} x_{k}\right)}_{(\text {C. } 7)} \\
& \times \underbrace{\frac{3 D_{k}}{4 \sqrt{2 \pi}\left(N-N_{R, k}\right)^{5 / 2}} \exp \left(-A_{k} x_{k}\right)}_{(\text {C. } 8)} \\
& -\underbrace{\left(\frac{A_{k}^{2}}{\sigma_{n, k}^{2} \sqrt{2 \pi} N_{S_{\max }^{3 / 2}}}-\frac{A_{k} D_{k}}{2 \sigma_{n, k}^{2}\left(N-N_{R, k}\right)^{3 / 2}} \exp \left(-A_{k} x_{k}\right)\right)^{2}} \\
& \text { (C.9) } \\
& =\frac{A_{k}^{2} D_{k}^{2}}{4 \pi \sigma_{n}^{4}\left(N-N_{R, k}\right)^{3}} \exp \left(-2 A_{k} x_{k}\right) \\
& +\frac{A_{k}^{3} D_{k}}{2 \pi \sigma_{n}^{4}\left(N-N_{R, k}\right)^{3 / 2} N_{S_{\max }^{3 / 2}}} \exp \left(-A_{k} x_{k}\right)-\frac{A_{k}^{4}}{2 \pi \sigma_{n}^{4} N_{S_{\max }}^{3}} .
\end{aligned}
$$

We re-arrange (C.12) to obtain

$$
\frac{2 A_{k}^{2}\left(N-N_{R, k}\right)^{3}}{N_{S_{\max }}^{3}} \exp \left(2 A_{k} x_{k}\right)-\frac{2 A_{k} D_{k}\left(N-N_{R, k}\right)^{3 / 2}}{N_{S_{\max }^{3 / 2}}} \exp \left(A_{k} x_{k}\right)-D_{k}^{2} \leq 0 .
$$

Unfortunately, (C.13) is a non-convex constraint. However, we can split it and bound each component to derive convex constraints. First, we upper-bound the positive term. Since $\left(N-N_{R, k}\right) \leq N_{S_{\max }}$, we can bound

$$
\frac{2 A_{k}^{2}\left(N-N_{R, k}\right)^{3}}{N_{S_{\max }}^{3}} \exp \left(2 A_{k} x_{k}\right) \leq A_{k}^{2} \exp \left(2 A_{k} x_{k}\right)+\frac{A_{k}^{2}\left(N-N_{R, k}\right)^{3}}{N_{S_{\max }}^{3}} \exp (E),
$$

where

$$
2 A_{k} x_{k}-E \leq 0
$$

is a convex constraint and $E$ is a tunable parameter that limits the maximum value of $\tau_{k}$. In practice, we choose $E=1$. Note that this form of bound enables a larger convex region than by simply using $\left(N-N_{R, k}\right) / N_{S_{\max }} \leq 1$. 
Next, we upper-bound the negative fraction in (C.13). Since $A_{k} x_{k}>0$, we bound

$$
-\frac{2 A_{k} D_{k}\left(N-N_{R, k}\right)^{3 / 2}}{N_{S_{\max }^{3 / 2}}^{3 / 2}} \exp \left(A_{k} x_{k}\right) \leq-\frac{2 A_{k} D_{k} N_{S_{\min }}^{3 / 2}}{N_{S_{\max }}^{3 / 2}} .
$$

Thus, we bound (C.13) with the convex bound

$$
A_{k}^{2} \exp \left(2 A_{k} x_{k}\right)+\frac{A_{k}^{2}\left(N-N_{R, k}\right)^{3}}{N_{S_{\max }}^{3}} \exp (E)-\frac{2 A_{k} D_{k} N_{S_{\min }}^{3 / 2}}{N_{S_{\max }}^{3 / 2}}-D_{k}^{2} \leq 0
$$

and by satisfying (C.17) and (C.15), we satisfy (C.3). Thus, under these additional constraints, (3.15) is convex. 


\section{Appendix D}

\section{Proof of Theorem 3}

$\bar{P}_{R, k}$ can be defined as

$$
\bar{P}_{R, k}=\mathbb{E}_{\left|g_{k}\right|}\left\{P_{R, k}\right\},
$$

where $\mathbb{E}_{x}\{\cdot\}$ is the expected value with respect to variable $x$. Since $\left|g_{k}\right|$ is Rayleighdistributed, $\left|g_{k}\right|^{2}$ has exponential probability distribution [28, App. A]

$$
p(r)=2 r \exp \left(-r^{2} / \sigma_{g, k}^{2}\right) / \sigma_{g, k}^{2}
$$

By performing the transformation of variables

$$
\lambda=\left|g_{k}\right| \sqrt{2 N_{R, k} / \sigma_{z, k}^{2}},
$$

we can write

$$
\bar{P}_{R, k}=\int_{0}^{\infty} \lambda w \exp \left(-\lambda^{2} w / 2\right) Q(\lambda) d \lambda,
$$

where $w=\sigma_{z, k}^{2} /\left(N_{R, k} \sigma_{g, k}^{2}\right)$. We apply the Craig representation of the $Q$-function (as in [18]),

$$
Q(x)=\int_{0}^{\frac{\pi}{2}} \exp \left(-x^{2} / \sin ^{2} \theta\right) d \theta,
$$

and a variable substitution to obtain

$$
\bar{P}_{R, k}=\frac{w}{\pi} \int_{0}^{\frac{\pi}{2}} \frac{d \theta}{w+1 / \sin ^{2} \theta} .
$$


We know that $1 \leq 1 / \sin ^{2} \theta \leq \infty$ and we can expect $w$ to be small, likely less than 1 , due to $N_{R, k}$. Therefore, we can approximate $w$ in the integral of (D.6) as 0 and upper-bound $\bar{P}_{R, k}$ by

$$
\bar{P}_{R, k} \leq \frac{w}{\pi} \int_{0}^{\frac{\pi}{2}} \sin ^{2} \theta d \theta
$$

Eq. (D.7) is easily solved to give the upper bound in(3.24). 


\section{Appendix E}

\section{Proof of Theorem 4}

The $(-1)^{i-M}$ term with the binomial coefficient in (4.1) is 1 for $i=M$ and the sign changes as $i$ increases. For $M \geq K-1$, the proof of (4.5) is trivial and no constraints are required. For $M<K-1$, if we can show how the combinations of products of $M+2 b-1$ terms (i.e., those with negative magnitude) cancel out the combinations of products of $M+2 b$ terms (i.e., those with positive magnitude), for $b \in\left\{1,2, \ldots,\left\lfloor\frac{K-M}{2}\right\rfloor\right\}$, then (4.5) is an upper bound. Thus, from (4.1) we must show when

$$
\begin{aligned}
\left(\begin{array}{c}
M+2 b-2 \\
2 b-1
\end{array}\right) \sum_{S_{K, M+2 b-1, j} \in \mathbf{S}_{K, M+2 b-1}} & \prod_{k=1}^{M+2 b-1} P_{f F C, k} \\
\geq & \left(\begin{array}{c}
M+2 b-1 \\
2 b
\end{array}\right) \sum_{S_{K, M+2 b, j} \in \mathbf{S}_{K, M+2 b}} \prod_{k=1}^{M+2 b} P_{f F C, k} .
\end{aligned}
$$

The left-hand side of (E.1) includes $\left(\begin{array}{c}K \\ M+2 b-1\end{array}\right)$ combinations of products of $M+$ $2 b-1$ probability terms, while the right-hand side includes $\left(\begin{array}{c}K \\ M+2 b\end{array}\right)$ combinations of products of $M+2 b$ terms. It is straightforward to show that

$$
\left(\begin{array}{c}
K \\
M+2 b
\end{array}\right)=\left(\begin{array}{c}
K \\
M+2 b-1
\end{array}\right) \frac{K-M-2 b+1}{M+2 b}
$$

so the right-hand side will have $\frac{K-M-2 b+1}{M+2 b}$ more products than the left-hand side. 
Comparing the coefficients, it is straightforward to show that

$$
\left(\begin{array}{c}
M+2 b-1 \\
2 b
\end{array}\right)=\left(\begin{array}{c}
M+2 b-2 \\
2 b-1
\end{array}\right) \frac{M+2 b-1}{2 b},
$$

so, by combining (E.2) and (E.3), we claim that the righthand side is effectively $\frac{K-M-2 b+1}{M+2 b} \cdot \frac{M+2 b-1}{2 b}$ times greater than the lefthand side, but with an additional probability for every combination. We wish to guarantee that the "extra" probability is small enough to satisfy (E.1).

As a related aside, consider the problem

$$
\begin{array}{ll}
\text { maximize } & \prod_{i}^{K} p_{i} \\
\text { subject to } & \sum_{i}^{K} p_{i}=T,
\end{array}
$$

where we assume that $0 \leq p_{i} \leq 1, \forall i$. Problem (E.4) has a quasiconcave objective function [22] and the optimal solution is $p_{1}=p_{2}=\ldots=p_{K}=T / K$.

The result of this aside is that a constraint on the mean value of a set of probability terms is an upper bound on the product of those probability terms. Thus, from (E.2) and (E.3), we satisfy (E.1) if

$$
\frac{\sum_{k=1}^{K} P_{f F C, k}}{K} \leq \frac{2 b M+4 b^{2}}{K(M-1)-M^{2}+2 M-1+2 b(K-2 M+2)-4 b^{2}},
$$

which is an increasing function of $b$. Thus, the constraint is tighest when $b=1$, which gives (4.7) to prove (4.5).

The proof of (4.6) is analogous to that of (4.5) and results in constraint (4.8). 